\title{
Polyphenol-Rich Dry Common Beans (Phaseolus vulgaris L.) and Their Health Benefits
}

\author{
Kumar Ganesan and Baojun $\mathrm{Xu}$ * \\ Food Science and Technology Program, Division of Science and Technology, Beijing Normal \\ University-Hong Kong Baptist University United International College, Zhuhai 519087, China; \\ kumarganesan@uic.edu.hk \\ * Correspondence: baojunxu@uic.edu.hk; Tel.: +86-756-3620-636; Fax: +86-756-3620-882
}

Received: 25 October 2017; Accepted: 2 November 2017; Published: 4 November 2017

\begin{abstract}
Polyphenols are plant metabolites with potent anti-oxidant properties, which help to reduce the effects of oxidative stress-induced dreaded diseases. The evidence demonstrated that dietary polyphenols are of emerging increasing scientific interest due to their role in the prevention of degenerative diseases in humans. Possible health beneficial effects of polyphenols are based on the human consumption and their bioavailability. Common beans (Phaseolus vulgaris L.) are a greater source of polyphenolic compounds with numerous health promoting properties. Polyphenol-rich dry common beans have potential effects on human health, and possess anti-oxidant, anti-diabetic, anti-obesity, anti-inflammatory and anti-mutagenic and anti-carcinogenic properties. Based on the studies, the current comprehensive review aims to provide up-to-date information on the nutritional compositions and health-promoting effect of polyphenol-rich common beans, which help to explore their therapeutic values for future clinical studies. Investigation of common beans and their impacts on human health were obtained from various library databases and electronic searches (Science Direct PubMed, and Google Scholar).
\end{abstract}

Keywords: polyphenols; Phaseolus vulgaris; anti-oxidants; degenerative diseases; health-promoting effects

\section{Introduction}

Plants synthesize secondary metabolites that often have widespread bioactivities, and are known as phytochemicals. Polyphenol is one of the phytochemicals containing large bioactive structural phenolic units. It has a wide range of classification and possesses various pharmacological and health-promoting effects [1]. Polyphenols are largely found in fruits, cereals, vegetables, food legumes, herbs, spices, nuts, wine, olive oil, tea, coffee, and chocolate. Polyphenols are classified into different groups based on the function of several phenyl rings, including flavonoids (flavones, flavonols, flavanones, isoflavones, anthocyanins, chalcones, dihydrochalcones, and catechins), phenolic acids (hydroxybenzoic hydroxyphenyl acetic, hydroxyphenyl pentanoic and hydroxyl cinnamic acids), stilbenes, and lignans [2]. The primary functions of polyphenols are as anti-oxidants involved in the prevention of degenerative diseases such as cancer and metabolic syndromes [2]. The health-promoting effects of polyphenols depend on the quantity consumed in the diet and their bioavailability. In addition, polyphenols are the active substances in many food legumes, which regulate the activity of a broad spectrum of cell receptors, enzymes and gene expression [3]. Animal experimental studies showed that polyphenol in common beans possess anti-oxidant properties and have various biological activities including anti-diabetic, anti-obesity, anti-inflammatory, antimicrobial, anticancer, hepatoprotective, cardioprotective, nephroprotective, neuroprotective, and osteoprotective [4-11]. 


\section{Common Beans and Their Health Benefits}

Common beans (Phaseolus vulgaris L.) are annual plants, cultivated in temperate and semitropical regions for their edible dry seeds that are variously called navy beans, kidney beans, red beans, black beans, pinto beans, and cranberry beans. They were first cultivated in Peru and Mexico around 8000 years ago and are now cultivated worldwide [12]. They belong to the family Fabaceae. In the temperate regions, the green leaves and immature pods are edible as vegetables. Dry beans are mainly consumed in low- and middle-class families as the large portion of the protein. In many parts of Asian, young leaves are consumed as a salad. The straw of the plant is normally used for fodder after beans are harvested. In 2010, the global production of dried beans was 25.42 million metric tons, and they were harvested on 32 million hectares. About $19.23 \%$ of the productions was in India followed by Myanmar (13.88\%), Brazil (12.42\%), USA (5.66\%), China (5.26\%), Mexico (4.52\%) and Tanzania (4.28\%) [12]. The production of dried beans worldwide in 2014 was 27.59 million metric tons, and they were harvested on 31.22 million hectares. About $16.85 \%$ of the productions was in Myanmar followed by India (14.89\%), Brazil (11.92\%), USA (4.74\%), Mexico (4.60\%), Tanzania (4.023\%) and China (3.84\%) [12]. Beans are known to be used for treating eczema, diabetes, diuretic, burns, acne, cardiac, bladder, carminative, dropsy, dysentery, emollient, hiccups, itchy, and rheumatism [13].

Common beans do not differ mostly in their nutritional compositions; they differ slightly in taste, texture and cooking times [14]. Navy beans are white in color, and were used in the U.S. Navy diet during the 19th century; hence, their name. They are small-sized, white-skinned, oval-shaped beans. Navy bean-containing diets exerted beneficial effects during experimental colitis by reducing inflammatory biomarkers both locally and systemically [15]. Emerging evidence supports the efficacy of navy beans in regulating serum cholesterol and lipid profiles, and inhibiting the incidence and recurrence of adenomatous polyps or precancerous growths, thereby preventing colorectal cancer [16,17]. Kidney beans are large-sized, firm textured, red/pink glossy skinned and kidney-shaped beans. They have the potential to reduce glycemic index in experimental diabetes [18] and the ability to attenuate colonic inflammation in healthy mice [19]. Red beans are small, soft red textured, oval-shaped beans. They exert an anti-inflammatory response [20] and have health-promoting potential with anti-fungal, immunomodulatory, anti-proliferative and apoptosis-inducing activities in tumor cells [21,22]. Black beans are known as turtle beans, which are sweet in taste, soft texture, medium-sized, and oval-shaped beans. These coats are an excellent source of anthocyanins and other phenolics with the potential to be used as natural food colorants with exceptional anti-diabetic potential [23]. Pinto beans are medium-sized, brown-skinned, oval-shaped beans. Hemagglutinins, defensins isolated from pinto beans, possess anti-fungal, anti-diabetic and anti-tumor activities [24,25]. Cranberry beans, also called Roman beans, are red creamy textured, medium-sized and oval-shaped beans. They are rich in phenolic compounds and non-digestible fermentable components, which may help alleviate experimental colitis and mitigate the severity of other gut barrier-associated pathologies [26].

Common beans play a vital role in the vegetarian diets and provide numerous health benefits connected with eating pattern [27]. They serve as a cost-effective source of nutrients. Health benefits of beans are generally acquired from direct attributes, including their high content of proteins, dietary fibers, low saturated fat content, vitamins, minerals, and phytochemicals, as well as replacement in the diet, when they substitute for animal products [28]. These replacements of meat and other animal products with beans are highly linked with enhanced animal welfare and the decrease in inputs of environmental resources [28]. Sufficient amounts of polyphenols in the dried beans act as potent anti-oxidants. Regular intake of these dried beans containing total and soluble fiber as well as resistant starches have reduced glycemic index in the human. Studies have also suggested that diets that include beans reduce low density lipoprotein (LDL), increase high density lipoprotein (HDL) levels and positively affect risk factors for metabolic syndrome, and thereby decrease the risk of cardiovascular diseases (CVD) obesity and diabetes [29]. The Food Habits in Later Life study conducted in Japanese, Greek and Australian populations have demonstrated that dried beans and other food 
legumes are the only foods linked with a reduced risk of mortality [30]. Hence, health-promoting effects are directly proportional to increased bean intake.

\section{Nutritional Compositions of Common Beans}

Dry common beans (fully matured and dried) are a rich source of proteins, starch, unsaturated fatty acids (linoleic acid), dietary fibers, vitamins and minerals that are considered as important food resources. These dry beans are normally soaked and cooked for few hours, and served as soups, stews, and meat dishes. Green beans (green immature pods) have greater quantities of vitamin $C$ and dietary fiber and are often sold as canned or frozen in the USA, while sold as fresh vegetables in China. Nutritional properties of the beans are highly linked to their measure of protein, and, to a smaller extent, their carbohydrate, vitamin, and mineral contents. The protein present in the bean types is different based on the cultivars, which ranges between $15 \%$ and $35 \%$. The predominant amino acids present in the dry beans are lysine (6.5-7.5 g/100 g protein) and tyrosine with phenylalanine (5.0-8.0 g/100 g protein) [31]. Consequently, the protein present in the beans meets the minimal need of human requirements endorsed by the World Health Organization and Food and Agriculture Organization. Thus, $100 \mathrm{~g}$ of dry common beans serve in human provides about $9-25 \mathrm{~g}$ of protein, which is almost $20 \%$ of the recommended daily consumption for a normal adult. In addition, the digestibility of the dry bean protein is almost $80 \%$ [32]. About 55-75 $\mathrm{g}$ of carbohydrates are present in $100 \mathrm{~g}$ of raw beans and predominant fraction in the bean is starch, constituting almost $50 \%$ of the seed weight. In addition, dietary fibers (14-19 g/100 g of raw) and oligosaccharides are significant quantities [33]. More than 50\% of fibers are insoluble, composed of pectins, pentosans, hemicellulose, cellulose, and lignin. The lipid fraction in the bean is about 1.5-6.5 $\mathrm{g}$ in $100 \mathrm{~g}$ of raw beans and is mainly composed of mono- and polyunsaturated fatty acids [34].

Dry beans contain biologically active phytochemicals, which are beneficial for human health [3]. While the beans contain huge quantities of protein, it is connected with anti-nutritional factors and other substances that are harmful to human health including polyphenols (including tannins), proteases, lectins, anti-vitamins, galacto-oligosaccharides, flatulence factors, allergens, and phytic acid [35]. Among the anti-nutritional factors, polyphenols are the primary contributors to reduce digestion of the bean in the human. They are highly active and can react with protein to cause impairment of the digestion. Tannins in the beans are potent and have the ability to bind with proteins by H-bonds, and thus prevent their digestion [36]. Boiling bean seeds is the common method of processing, and results in a decrease in the polyphenol content and reduces anti-nutritional factors [37]. The germination mechanism is also improving the levels of free amino acids, nutritional quality, and decreases the anti-nutritional factors [38]. The nutritional compositions of common beans are listed in Table 1.

Common beans have the highest source of protein and other dietary nutrition by complementing other foods, including meat, wheat, cereals and other food legumes [39]. The protein content of beans is almost equal to that of meat, ranging between $20 \%$ and $30 \%[40,41]$. The primary protein fractions in beans are globulin (50-70\%) and albumin (10\%). Based on the sedimentation coefficient, the protein fractions of globulins are classified into 7S and 11S; both are natural oligomers [42,43]. The 7S fraction is normally referred to as phaseolin, an active glycoprotein consisting of about $50 \%$ of the total bean nitrogen, whereas the $11 \mathrm{~S}$ globulin fraction is only $10 \%$ [44]. Prolamine and glutelin are also present as in minor quantities [45]. Common beans contain the highest ranges of glutelins (20-30\%) when compared with other food legumes (7-15\%) [46,47]. Similar to other food legumes, common beans contain a greater amount of essential amino acids, including lysine, which is deficient in most cereals. Beans are an excellent source of micronutrients such as minerals and vitamins and observed superior to cereals [48]. They have the highest vitamin and mineral contents comparing to all legumes [49]. Similar to other food legume seeds, dried beans contain numerous bioactive compounds such as galacto-oligosaccharides, protease inhibitors, lectins, phytates, oxalates and phenolic-rich substances that play crucial metabolic function in humans and animals [50]. Based on the diet quality, some of 
these substances have been known as anti-nutritional factors. These substances can reduce protein digestibility, diminish nutrient absorption and mineral bioavailability, which may cause flatulence in human [51]. However, these anti-nutritional factors have antioxidant and prebiotic activities, and protect DNA damage against various cancers [52-56]. Hence, common beans are nutritionally complementary with respect to essential amino acids, vitamins, minerals, and anti-nutritional factors, and the consumption of common beans could alleviate deficiency status, ensuring in the balanced $\operatorname{diet}[47,57]$.

Table 1. Nutritional compositions of common beans in $100 \mathrm{~g}$ of edible portion [58-60].

\begin{tabular}{|c|c|c|c|c|c|c|c|}
\hline Nutrient & Units & $\begin{array}{l}\text { Navy } \\
\text { Beans }\end{array}$ & $\begin{array}{l}\text { Kidney } \\
\text { Beans }\end{array}$ & $\begin{array}{c}\text { Red } \\
\text { Beans }\end{array}$ & $\begin{array}{l}\text { Black } \\
\text { Beans }\end{array}$ & $\begin{array}{l}\text { Pinto } \\
\text { Beans }\end{array}$ & $\begin{array}{c}\text { Cranberry } \\
\text { Beans }\end{array}$ \\
\hline Energy & kcal & 92 & 92 & 167 & 464 & 500 & 257 \\
\hline Protein & $\mathrm{g}$ & 6.15 & 5.38 & 22.22 & 14.29 & 10.71 & 22.86 \\
\hline Total lipid (fat) & $\mathrm{g}$ & 0.00 & 0.38 & 0.00 & 21.43 & 21.43 & 0.00 \\
\hline Carbohydrate & $\mathrm{g}$ & 16.15 & 20.77 & 63.89 & 57.14 & 60.71 & 60.00 \\
\hline Total dietary fiber & $\mathrm{g}$ & 4.6 & 5.4 & 44.4 & 14.3 & 10.7 & 25.7 \\
\hline Total sugars & $\mathrm{g}$ & 0.00 & 0.77 & 2.78 & 3.57 & 3.57 & 2.86 \\
\hline Resistant starch & $\mathrm{g}$ & 4.2 & 2.0 & 3.8 & 1.7 & 1.9 & 2.6 \\
\hline \multicolumn{8}{|c|}{ Minerals } \\
\hline Calcium & $\mathrm{mg}$ & 62 & 46 & 167 & 143 & 71 & 114 \\
\hline Iron & $\mathrm{mg}$ & 1.38 & 1.38 & 7.29 & 3.86 & 2.57 & 5.1 \\
\hline Potassium & $\mathrm{mg}$ & 300 & 268 & 222 & 279 & 96 & 265 \\
\hline Magnesium & $\mathrm{mg}$ & 48 & 37 & 44 & 60 & 43 & 39 \\
\hline Sodium & $\mathrm{mg}$ & 108 & 8 & 69 & 286 & 286 & 10 \\
\hline \multicolumn{8}{|c|}{ Vitamins } \\
\hline Vitamin C & $\mathrm{mg}$ & 0.9 & 0.9 & 0.0 & 0.0 & 0.0 & 0.0 \\
\hline Folate & $\mu \mathrm{g}$ & 127 & 115 & 140 & 128 & 147 & 124 \\
\hline \multicolumn{8}{|c|}{ Lipids } \\
\hline Total saturated & $\mathrm{g}$ & 0.000 & 0.000 & 0.000 & 1.790 & 1.790 & 0.000 \\
\hline Total monounsaturated fatty acids & $\mathrm{g}$ & 0.000 & 0.000 & 0.000 & 14.290 & 14.290 & 0.000 \\
\hline Total polyunsaturated fatty acids & $\mathrm{g}$ & 0.000 & 0.000 & 0.000 & 5.360 & 5.360 & 0.000 \\
\hline Polyphenol & $\begin{array}{l}\text { mg of gallic } \\
\text { acid equiv/g }\end{array}$ & 12.47 & 14.14 & 13.68 & 12.60 & 12.52 & 11.73 \\
\hline Flavonoids & $\begin{array}{l}\text { mg of rutin } \\
\text { equiv/g }\end{array}$ & 1.78 & 2.59 & 1.55 & 1.28 & 0.98 & 1.65 \\
\hline
\end{tabular}

\section{Polyphenols in Common Beans}

The dry bean contains plenty of polyphenols. Studies have demonstrated that phenolic compounds are predominantly located in the seed coat of the bean than in the cotyledon and testa [61]. The content of the phenolic compound is about $145 \mathrm{mg} / \mathrm{g}$ and represents about $11 \%$ of the total seed [62]. The phenolic compounds in the seeds are flavones, monomers, and oligomers of flavanols, flavanones, isoflavonoids, anthocyanins, chalcones, and dihydrochalcones [61,63-66]. However, the phenolic acids and non-flavonoid phenolic compounds (hydroxybenzoic and hydroxycinnamic acid) are mainly found in cotyledons of the bean [67]. Based on their chemical structure, they are a highly diverse group ranging from simple molecules such as phenolic acids to complex polymers such as tannins and lignin [68]. The testa of the beans contains greater quantities of proanthocyanidins and anthocyanins [61]. Condensed tannins (10.65 mg catechin equivalents/g) and cyanidin 3-glucoside (3.75 mg catechin equivalents/g) are also mainly present in seed coats of the bean $[69,70]$. These phenolic compounds are generally varied, based on the seed coat color pattern and types of the cultivar of the beans. The color of the seed coat is based on the presence of polyphenols including anthocyanins, flavonols glucosides, and condensed tannins. Dark-colored beans normally have the highest anthocyanins content [71]. In addition, red, black and pink-colored varieties confer color to the bean seed coat due to their anthocyanins. The colors of light yellow or pink spot of the seed coat are generally based on the presence of condensed tannins [70]. 
Phenolic compounds isolation and characterization were initiated at early 1960, and four anthocyanin pigments (delphinidin 3-glucoside, petunidin 3-glucoside, malvidin 3-glucoside and 3,5-diglucosides) were extracted from the seed coat of black violet beans [72]. Later, anthocyanins, flavonols, and tannins from the different varieties of kidney beans were isolated and characterized by many researchers [73-76]. Studies have further demonstrated that wild and weedy Mexican beans are rich in anthocyanins (delphinidin, delphinidin 3-glucoside, petunidin, petunidin 3-glucoside, cyanidin, malvidin, malvidin 3-glucoside, pelargonidin and peonidin), present in 62 Mexican wild-type varieties [77-79]. Raw and cooked beans contain predominant quantities of flavonoids including quercetin, myricetin, cynidine, procyanidin, naringenin, catechin, hesperetin and kaempferol [61]. The significant glucosides of the flavonoids are apigenin 7-O-glucoside, quercetin 3-O-glucoside, myricetin 3-O-glucoside, naringenin 7-O-glucoside, quercetin 4-O-galactoside and kaempferol 3-O-glucoside [80]. Variations in the flavonoids and its glucosides were observed in different bean varieties. Kaempferol and its 3-O-glucosides are primarily found in pinto beans; diglucosides of kaempferol and quercetin are found in dark red kidney beans; 3-O-glucosides of malvidin, petunidin, and delphinidin are present in black beans; quercetin 3-O-glucoside and its malonates are found in trace quantity in light red kidney beans; and kaempferol monoglucoside, kaempferol 3-O-glucoside, and kaempferol 3-O-xylosyl glucoside are found in Italian beans $[71,81]$.

Lima et al. [82] and Guajardo-Flores et al. [80] have analyzed in Brazilian beans containing non-glycosylated forms of isoflavonoids including daidzein $(0.0082-0.1291 \mathrm{mg} / \mathrm{g})$ and genistein $(0.0026-0.0097 \mathrm{mg} / \mathrm{g})$. Among them, black type of beans showed the highest concentrations of isoflavonoids and daidzein was the predominant compound. Raw and cooked beans contain phenolic acids, which may be derived from benzoic acid (vanillic, $p$-hydroxybenzoic, and gallic acids) and those derived from cinnamic acid (ferulic, $p$-coumaric, and chlorogenic acids). Among them, ferulic acid is the predominant phenolic acid on the dry common beans [78]. Studies have demonstrated that cooking of common beans at high temperature does not change the content of phenolic acids [83]. Raw common beans contain $p$-hydroxybenzoic acid $(0.0045-0.0086 \mathrm{mg} / \mathrm{g})$, vanillic acid $(0.0052-0.0166 \mathrm{mg} / \mathrm{g})$, coumaric acid $(0.0032-0.0068 \mathrm{mg} / \mathrm{g})$, and ferulic acid $(0.0017-0.0036 \mathrm{mg} / \mathrm{g})$ [84]. The polyphenols present in the common beans are illustrated in Table 2. 
Table 2. List of polyphenols in the common beans.

\begin{tabular}{|c|c|c|c|c|}
\hline Bean Name & Polyphenol Class & Polyphenol Sub-Class & Compound Name & References \\
\hline Dark bean & Flavonoids & Anthocyanins & $\begin{array}{l}\text { Cyanidin 3-O-glucoside, pelargonidin 3-O-glucoside, } \\
\text { petunidin-3-O- } \beta \text {-glucopyranoside, malvidin 3-O-glucoside, } \\
\text { delphinidin acetyl-glucoside, pelargonidin acetyl glucoside, } \\
\text { pelargonidin 3-O-malonyl glucoside, petunidin feruloyl glucose }\end{array}$ & [61] \\
\hline $\begin{array}{l}\text { Wild and weedy Mexican bean, pinto and } \\
\text { black beans }\end{array}$ & Flavonoids & Anthocyanins & Peonidin, pelargonidin, cyanidin & {$[78,85]$} \\
\hline Dark bean, Wild, and weedy Mexican bean & Flavonoids & Anthocyanins & Delphinidin 3-O-glucoside & {$[61,78]$} \\
\hline $\begin{array}{l}\text { Alubia, black, cranberry, dark red kidney, } \\
\text { great northern, light red kidney, navy, pink, } \\
\text { pinto, and small red }\end{array}$ & Flavonoids & Anthocyanins & Petunidin 3-O-(6"-acetyl-glucoside) & {$[71,81]$} \\
\hline Dark and kidney bean, zolfino landraces & Flavonoids & Anthocyanins & Pelargonidin 3,5-O-diglucoside & {$[45,61]$} \\
\hline $\begin{array}{l}\text { Alubia, black, cranberry, dark red kidney, } \\
\text { great northern, light red kidney, navy, pink, } \\
\text { pinto, and small red }\end{array}$ & Flavonoids & Anthocyanins & Delphinidin 3-O-glucosyl-glucoside & {$[71,86]$} \\
\hline Dark bean & Flavonoids & Flavanols & $\begin{array}{c}\text { (+)-Catechin, (-)-epicatechin, (+)-gallocatechin, procyanidin dimer, } \\
\text { (-)-epigallocatechin, Procyanidin dimer B2, procyanidin dimer B3, } \\
\text { procyanidin dimer B4, procyanidin trimer, procyanidin trimer EEC, } \\
\text { naringenin 7-glucoside }\end{array}$ & [61] \\
\hline Dark bean & Flavonoids & Flavanones & $\begin{array}{c}\text { Naringenin, hesperetin, naringin, naringenin 7-O-rutinoside, } \\
\text { naringenin 7-O-glucoside, naringenin-7-methyl ether 2, hesperetin } \\
3^{\prime}-O \text {-glucuronide, hesperetin 7-O-glucuronide, hesperetin } \\
3^{\prime}, 7-O \text {-diglucuronide, hesperetin 5,7-O-diglucuronide, hesperetin } \\
\text { 7-O-rutinoside }\end{array}$ & {$[61]$} \\
\hline Dark bean & Flavonoids & Flavones & Apigenin, apigenin 7-O-glucoside & [61] \\
\hline Brazilian bean & Flavonoids & Flavones & Chrysin & [66] \\
\hline Dark bean, Brazilian bean, Mexican bean & Flavonoids & Flavonols & Kaempferol & {$[35,61,66]$} \\
\hline Dark bean, Brazilian bean, Mexican bean & Flavonoids & Flavonols & Quercetin & {$[35,61,66]$} \\
\hline Dark bean, and Brazilian bean & Flavonoids & Flavonols & $\begin{array}{c}\text { Quercetin 3-O-galactoside, Quercetin 3-O-glucoside, Quercetin } \\
\text { 3-O-rutinoside, Myricetin, Myricetin 3-O-glucoside, Myricetin } \\
\text { 3-O-rhamnoside, Kaempferol 3-O-glucoside, Kaempferol } \\
\text { 3-O-rutinoside }\end{array}$ & {$[61,66]$} \\
\hline
\end{tabular}


Table 2. Cont

\begin{tabular}{|c|c|c|c|c|}
\hline Bean Name & Polyphenol Class & Polyphenol Sub-Class & Compound Name & References \\
\hline Pinto beans, zolfino landraces & Flavonoids & Flavonols & Kaempferol 3-O-glucosylxylose & {$[63,87]$} \\
\hline $\begin{array}{l}\text { Alubia, black, cranberry, dark red kidney, } \\
\text { great northern, light red kidney, navy, pink, } \\
\text { pinto, and small red }\end{array}$ & Flavonoids & Flavonols & Kaempferol 3-O-xylosyl-glucoside & {$[71,81]$} \\
\hline Pinto beans & Flavonoids & Flavonols & Kaempferol 3-O-acetyl-glucoside & [63] \\
\hline Dark bean, Brazilian bean & Flavonoids & Isoflavonoids & Daidzein & {$[61,66]$} \\
\hline Dark bean, Brazilian bean & Flavonoids & Isoflavonoids & Genistein & {$[61,66]$} \\
\hline Dark bean & Flavonoids & Isoflavonoids & Biochanin A & [61] \\
\hline Pinto and black beans & Flavonoids & Isoflavonoids & Glycitein & [85] \\
\hline Dark bean & Flavonoids & Isoflavonoids & Dihydrogenistein & [61] \\
\hline Brazilian bean & Polyphenols & Polyphenols & Coumestrol & [66] \\
\hline $\begin{array}{l}\text { Dark bean, pinto and black beans, } \\
\text { Mexican bean }\end{array}$ & Phenolic acids & Hydroxybenzoic acids & Protocatechuic acid & {$[35,61,85]$} \\
\hline Dark bean & Phenolic acids & Hydroxybenzoic acids & Gallic acid & [61] \\
\hline Mexican bean & Phenolic acids & Hydroxybenzoic acids & Vanillic acid & [35] \\
\hline Dark bean, Mexican bean & Phenolic acids & Hydroxycinnamic acids & $p$-Coumaric acid & {$[35,61]$} \\
\hline Pinto and black beans & Phenolic acids & Hydroxycinnamic acids & Caffeic acid & [85] \\
\hline Dark bean, Mexican bean & Phenolic acids & Hydroxycinnamic acids & Ferulic acid & {$[35,61]$} \\
\hline Dark bean & Phenolic acids & Hydroxycinnamic acids & Sinapic acid, Ferulic acid 4-glucoside & [61] \\
\hline Dark bean & Stilbenes & Stilbenes & trans-Resveratrol, resveratrol 3-O-glucoside & [61] \\
\hline
\end{tabular}




\section{Health Promoting Effects of Polyphenol-Rich Dry Beans}

The consumption of dry common bean has been greatly connected with many physiological and health promoting effects such as prevention of cardiovascular diseases, obesity, diabetes mellitus and cancers [86,88]. The anti-oxidant properties of polyphenol lie in their ability to neutralize free radicals and the chelation of transition metals, thus they counteract the initiation and propagation of oxidative processes [83]. Health promoting effects of polyphenol-rich dry common beans are illustrated in Figure 1.

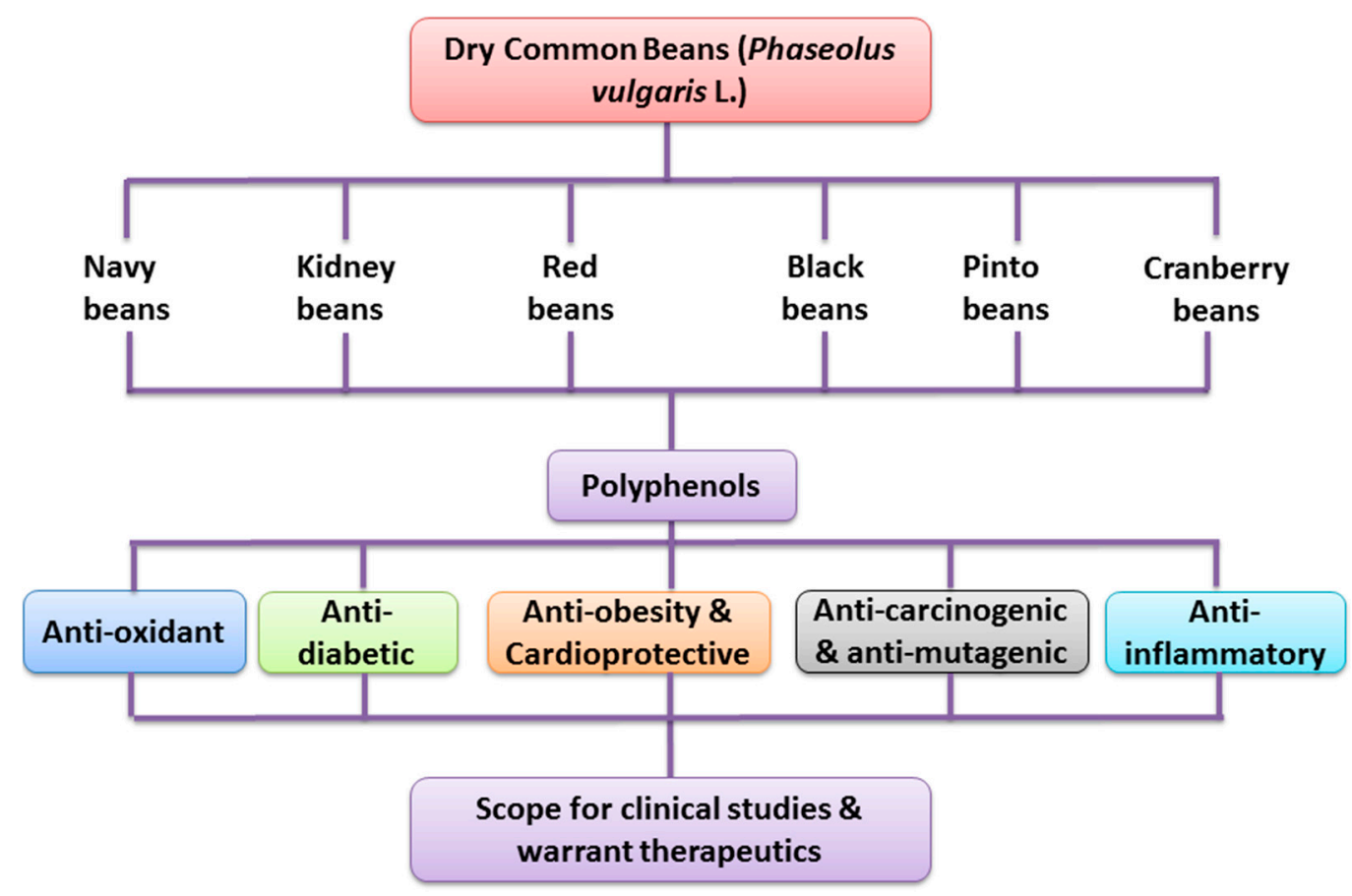

Figure 1. Health promoting effects of polyphenol-rich dry common beans.

\subsection{Anti-Oxidant Activity}

The dry common beans have excellent anti-oxidant activities because of its phenolic acids, flavonoids, stilbenes, and tannins. These anti-oxidant activities are primarily due to the reducing capacity of polyphenols as they play vital functions in neutralizing free radicals and scavenging radicals or suppressing lipid peroxidation [89]. In addition, polyphenols involve chelation of metal ions, causing impairment/cessation of oxidative mechanisms. Generally, the anti-oxidant activity is elevated during digestion and absorption of the common beans in the intestine. Normally, phenolic compounds are released higher in the stomach due to its acidic environment, and the acid medium and enzyme-mediated hydrolysis facilitate the higher solubility of polyphenols along with starch and proteins [65]. Common beans containing polyphenols have demonstrated the highest total anti-oxidant capacity measured by in vitro methods of 2,2'-Diphenyl-1-picrylhydrazyl (DPPH), $\beta$-carotene bleaching, ferric reducing anti-oxidant power, oxygen radical absorbing capacity, Trolox equivalent anti-oxidant capacity, and total radical-trapping anti-oxidant parameters [63,64,80,85,90-93]. Animal studies have also confirmed that common beans possess the highest anti-oxidant capacity, as measured in various biochemical parameters including thiobarbituric acid reactive substances (TBARS), hydroperoxides, glutathione (GSH), superoxide dismutase (SOD), catalase (CAT), glutathione reductase (GR), glutathione peroxidase (GPx), and glutathione S-transferase (GST) $[26,61,93,94]$. In vitro and in vivo studies of common beans exerting anti-oxidant activity are summarized in Table 3. 
Table 3. Summary of in vitro, in vivo and clinical studies on health-promoting effects of polyphenol-rich common beans (Phaseolus vulgaris L.).

\begin{tabular}{|c|c|c|c|c|c|c|}
\hline Bean Name & Polyphenols Names & Model/Subjects & Dosage & $\begin{array}{l}\text { Experimental } \\
\text { Period }\end{array}$ & Activities & References \\
\hline Kidney bean & $\begin{array}{c}p \text {-coumaric, ferulic and sinapic acids, } \\
\text { quercetin, kaempferol, procyanidins } \\
\text { B-2 and B-3 and tannins }\end{array}$ & $\begin{array}{c}\text { Brochothrix thermosphacta, Staphylococcus aureus, } \\
\text { Listeria monocytogenes Scott A, Salmonella typhimurium, } \\
\text { E. coli O157: H7, Pseudomonas fragi, } \\
\text { and Lactobacillusplantarum }\end{array}$ & 62.5 to $500 \mu \mathrm{g} / \mathrm{mL}$ & $36-48 \mathrm{~h}$ & Anti-bacterial activity & [95] \\
\hline Perla black bean & $\begin{array}{c}\text { Delphinidin 3-O-glucoside, petunidin } \\
\text { 3-O-glucoside and malvidin } \\
\text { 3-O-glucoside }\end{array}$ & $\begin{array}{l}\text { Bacterial strain: Pseudomonas aeruginosa, Proteus } \\
\text { vulgaris, Klebsiella oxytoca, Enterococcus faecalis, } \\
\text { Staphylococcus aureus, Staphylococcus epidermidis and } \\
\text { Listeria monocytogenes; Parasitic strain: Giardia lamblia, } \\
\text { Entamoeba hystolitica and Trichomonas vaginalis }\end{array}$ & $\begin{array}{l}0.05,0.5 \text { and } 5.0 \\
\mathrm{mg} / \text { disk }\end{array}$ & $36-48 \mathrm{~h}$ & $\begin{array}{l}\text { Anti-bacterial and } \\
\text { anti-parasitic activity }\end{array}$ & [96] \\
\hline $\begin{array}{l}\text { Peruvian and } \\
\text { Brazilian bean }\end{array}$ & Chlorogenic and caffeic acid & In vitro & $50 \mu \mathrm{L}$ & $24 \mathrm{~h}$ & $\begin{array}{l}\text { Anti-diabetic and } \\
\text { anti-hypertensive } \\
\text { activity }\end{array}$ & [97] \\
\hline Brown bean & Total phenolics & Human (16)-randomized crossover design & $100 \mathrm{~g} / \mathrm{bw} /$ p.o. & 30 days & $\begin{array}{l}\text { Anti-diabetic and } \\
\text { anti-obesity activity }\end{array}$ & [98] \\
\hline Kidney bean & Total phenolics and anthocyanins & $\begin{array}{l}\text { Human with overweight subjects (39)-A randomized, } \\
\text { double-blind, placebo-controlled clinical trial }\end{array}$ & $50 \mathrm{~g} / \mathrm{bw} /$ p.o. & 60 days & $\begin{array}{l}\text { Anti-diabetic and } \\
\text { anti-obesity activity }\end{array}$ & [99] \\
\hline Navy bean & Total phenolics and anthocyanins & 3T3-L1 adipocytes & $50 \mathrm{~g} / \mathrm{bw} /$ p.o. & 60 days & $\begin{array}{l}\text { Anti-diabetic and } \\
\text { anti-obesity activity }\end{array}$ & {$[100]$} \\
\hline Pinto bean & $\begin{array}{l}\text { Delphinidin glucoside, petunidin } \\
\text { glucoside, malvidin glucoside, } \\
\text { anthocyanins, catechin, myricetin } \\
\text { 3-O-arabinoside, epicatechin, vanillic } \\
\text { acid, syringic acid and } O \text {-coumaric acid }\end{array}$ & in vitro & $50 \mu \mathrm{L}$ & $24 \mathrm{~h}$ & Anti-diabetic activity & {$[101]$} \\
\hline Kidney bean & $\begin{array}{l}\text { Phenolic acids and bioactive peptide } \\
\text { fractions }\end{array}$ & In vitro & $\begin{array}{l}<1,1-3.5,3.5-5,5-10 \\
\text { and } 10 \mathrm{kDa}\end{array}$ & $24 \mathrm{~h}$ & Anti-diabetic activity & [102] \\
\hline Black bean & $\begin{array}{l}\text { Phenolic acids and bioactive peptide } \\
\text { fractions }\end{array}$ & In vitro & 10.20 to $0.34 \mathrm{mg}$ & $24 \mathrm{~h}$ & Anti-diabetic activity & [23] \\
\hline Black bean & $\begin{array}{l}\text { Delphinidin-3-O-glucoside, } \\
\text { petunidin-3-O-glucoside, } \\
\text { and malvidin-3-O-glucoside }\end{array}$ & Caco- 2 cells & $\begin{array}{c}\text { Anthocyanin } \\
\text { solutions }(1 \mathrm{mg} / \mathrm{mL}), \\
\text { purified anthocyanins } \\
(100 \mu \mathrm{M} \text { malvidin, } \\
100 \mu \mathrm{M} \text { delphinidin }) \\
\text { or phloretin }(100 \mu \mathrm{M}) \text {. }\end{array}$ & $24-36 \mathrm{~h}$ & Anti-diabetic activity & [23] \\
\hline
\end{tabular}


Table 3. Cont

\begin{tabular}{|c|c|c|c|c|c|c|}
\hline Bean Name & Polyphenols Names & Model/Subjects & Dosage & $\begin{array}{l}\text { Experimental } \\
\text { Period }\end{array}$ & Activities & References \\
\hline Black bean & $\begin{array}{c}\text { Total phenolic, tannins and } \\
\text { anthocyanins }\end{array}$ & Human (56)-diabetic patients & $100 \mathrm{~g}$ of black bean & 3 months & Anti-diabetic activity & [103] \\
\hline Kidney bean & $\begin{array}{l}\text { Total phenolic, tannins and } \\
\text { anthocyanins }\end{array}$ & Wistar albino rats & $200 \mathrm{mg} / \mathrm{kg} \mathrm{bw}$ & 30 days & Anti-diabetic activity & [104] \\
\hline Black bean & $\begin{array}{c}\text { Total phenolic, tannins and } \\
\text { anthocyanins }\end{array}$ & Wistar albino rats & $200 \mathrm{mg} / \mathrm{kg} \mathrm{bw}$ & 45 days & Anti-diabetic activity & [105] \\
\hline Pinto bean & Total phenolics & $\begin{array}{l}\text { Human (12)-randomized, double-blind, } \\
\text { placebo-controlled study }\end{array}$ & 100 g/bw/p.o. & $3 \mathrm{~h}$ & Anti-diabetic activity & [106] \\
\hline Navy bean & Total phenolics & $\begin{array}{l}\text { Human with diabetes (17)-randomized } \\
4 \times 4 \text { crossover trial }\end{array}$ & $50 \mathrm{~g} / \mathrm{bw} / \mathrm{p} . \mathrm{o}$ & $24 \mathrm{~h}$ & Anti-diabetic activity & {$[107]$} \\
\hline Black bean & Total phenolics and anthocyanins & In vitro & $100 \mu \mathrm{g}$ & $24 \mathrm{~h}$ & Anti-diabetic activity & [108] \\
\hline White kidney bean & Total phenolics & Wistar albino rats & 50 mg/kg bw/p.o. & 7 days & Anti-diabetic activity & {$[109,110]$} \\
\hline Black bean & $\begin{array}{l}\text { Phenolic acids and bioactive } \\
\text { peptide fractions }\end{array}$ & In vitro & $10.20-0.34 \mathrm{mg}$ & $24 \mathrm{~h}$ & $\begin{array}{c}\text { Anti-diabetic, } \\
\text { and anti-hypertensive } \\
\text { activities }\end{array}$ & [101] \\
\hline Zolfino landrace & Phenolic acids & in vitro & $700 \mu \mathrm{L}$ & $24 \mathrm{~h}$ & $\begin{array}{c}\text { Anti-diabetic, } \\
\text { anti-oxidant and } \\
\text { anti-inflammatory } \\
\text { activities }\end{array}$ & [111] \\
\hline Kidney bean & $\begin{array}{l}\text { Phenolic acids (chlorogenic acid, gallic } \\
\text { acid, } p \text {-hydroxy benzoic acid, caffeic } \\
\text { acid, protocatechuic acid, } p \text {-coumaric } \\
\text { acid, rosmarinic acid, ferulic acid, } \\
\text { sinapic acid and ellagic acid) and } \\
\text { flavonoids (epicatechin, cate chin, } \\
\text { gallocatechin gallate, epigallocatechin } \\
\text { gallate, quercetin, hesperidin, } \\
\text { and rutin) }\end{array}$ & Male Wistar rats & $\begin{array}{l}0.4,0.8 \text { and } 1.2 \mathrm{~g} / \mathrm{kg} \\
\text { bw } / \text { p.o. for } 6 \text { weeks }\end{array}$ & 21 days & $\begin{array}{c}\text { Anti-diabetic, } \\
\text { hypolipidemic and } \\
\text { cardioprotective } \\
\text { activity }\end{array}$ & [112] \\
\hline Kidney bean & Lectins and polyphenol & $\begin{array}{c}\text { Fusarium oxysporum, Coprinus comatus, } \\
\text { and Rhizoctonia solani }\end{array}$ & $20-200 \mu \mathrm{g} / \mathrm{mL}$ & $24 \mathrm{~h}$ & Antifungal activity & [113] \\
\hline Kidney bean & Total phenolics & Sprague-Dawley rats & $\begin{array}{c}0,7.5 \%, 15 \%, 30 \% \text { or } \\
60 \% w / w\end{array}$ & 7 days & $\begin{array}{l}\text { Anti-hepatotoxic } \\
\text { effect }\end{array}$ & [114] \\
\hline
\end{tabular}


Table 3. Cont

\begin{tabular}{|c|c|c|c|c|c|c|}
\hline Bean Name & Polyphenols Names & Model/Subjects & Dosage & $\begin{array}{l}\text { Experimental } \\
\text { Period }\end{array}$ & Activities & References \\
\hline White and red bean & $\begin{array}{c}\text { Ferulic, coumaric, Sinapic acid, } \\
\text { Catechin, Malvidin 6-O-glucoside, } \\
\text { Quercetin, }\end{array}$ & Macrophages cell line RAW 264.7 & $20 \mu \mathrm{L}$ & $36-48 \mathrm{~h}$ & $\begin{array}{l}\text { Anti-inflammatory } \\
\text { activity }\end{array}$ & [115] \\
\hline $\begin{array}{l}\text { Navy and pinto } \\
\text { bean }\end{array}$ & $\begin{array}{l}\text { Phenolic acids and bioactive peptide } \\
\text { fractions }\end{array}$ & RAW 264.7 macrophages & $\begin{array}{l}1-3.5,3.5-5,5-10 \\
\text { and } 10 \mathrm{kDa}\end{array}$ & $36-48 \mathrm{~h}$ & $\begin{array}{l}\text { Anti-inflammatory } \\
\text { activity }\end{array}$ & [102] \\
\hline $\begin{array}{l}\text { Black, navy, kidney } \\
\text { and pinto bean }\end{array}$ & $(+)$-catechin & Salmonella typhimurium strains TA98 and TA100 & $\begin{array}{c}2.5,5,10,12.5,15 \text { and } \\
25 \mu \mathrm{g}\end{array}$ & $24 \mathrm{~h}$ & $\begin{array}{l}\text { Anti-mutagenic } \\
\text { activity }\end{array}$ & [62] \\
\hline $\begin{array}{l}\text { Black and kidney } \\
\text { beans }\end{array}$ & $\begin{array}{l}\text { Quercetin, kaempferol, } p \text {-coumaric } \\
\text { acid, ferulic acid, } p \text {-hydroxybenzoic } \\
\text { acid, and vanillic acid }\end{array}$ & $\begin{array}{l}\text { Sprague-Dawley rats and a diet-induced obesity } \\
\text { model in C57Bl/ } 6 \text { mice }\end{array}$ & $\begin{array}{l}7.5 \%, 15 \%, 30 \% \text { or } \\
\quad 60 \% w / w\end{array}$ & 7 days & Anti-obesity activity & [116] \\
\hline Black bean & Total phenolics & In vitro & $50-200 \mu \mathrm{L}$ & $24 \mathrm{~h}$ & $\begin{array}{l}\text { Anti-oxidant and } \\
\text { anti-inflammatory } \\
\text { activities }\end{array}$ & [117] \\
\hline Kidney bean & Flavonoids & HMEC-1 line & $0.7 \mathrm{mg}$ & $36-48 \mathrm{~h}$ & $\begin{array}{l}\text { Anti-oxidant and } \\
\text { anti-inflammatory } \\
\text { activities }\end{array}$ & [118] \\
\hline Black bean & $\begin{array}{l}\text { Total phenolic, tannins and } \\
\text { anthocyanins }\end{array}$ & Human (12)-randomized, controlled, crossover trial & $\begin{array}{l}100 \mathrm{~g} \text { of black bean } \\
\text { meal and soup }\end{array}$ & 3 days & $\begin{array}{l}\text { Anti-oxidant and } \\
\text { anti-inflammatory } \\
\text { activities }\end{array}$ & [119] \\
\hline $\begin{array}{l}\text { Navy and black } \\
\text { bean }\end{array}$ & $\begin{array}{l}\text { Phenolic acids, flavonoids, } \\
\text { and anthocyanins }\end{array}$ & C57BL/6 mice & $\begin{array}{l}20 \% \text { navy bean or } \\
\text { black bean/p.o. }\end{array}$ & 2 weeks & $\begin{array}{l}\text { Anti-oxidant and } \\
\text { anti-inflammatory } \\
\text { activities }\end{array}$ & [15] \\
\hline $\begin{array}{l}\text { White and dark } \\
\text { kidney beans }\end{array}$ & $\begin{array}{l}\text { Phenolic acids, flavonoids, } \\
\text { and anthocyanins }\end{array}$ & C57BL/6 mice & $\begin{array}{l}20 \% \text { navy bean or } \\
\text { black bean/p.o. }\end{array}$ & 2 weeks & $\begin{array}{l}\text { Anti-oxidant and } \\
\text { anti-inflammatory } \\
\text { activities }\end{array}$ & [19] \\
\hline Cranberry bean & $\begin{array}{l}\text { Phenolic acids, flavonoids, } \\
\text { and anthocyanins }\end{array}$ & C57BL/6 mice & $\begin{array}{l}20 \% \text { navy bean or } \\
\text { black bean/p.o. }\end{array}$ & 2 weeks & $\begin{array}{l}\text { Anti-oxidant and } \\
\text { anti-inflammatory } \\
\text { activities }\end{array}$ & {$[26]$} \\
\hline
\end{tabular}


Table 3. Cont

\begin{tabular}{|c|c|c|c|c|c|c|}
\hline Bean Name & Polyphenols Names & Model/Subjects & Dosage & $\begin{array}{l}\text { Experimental } \\
\text { Period }\end{array}$ & Activities & References \\
\hline $\begin{array}{l}\text { Navy and black } \\
\text { beans }\end{array}$ & $\begin{array}{l}\text { Phenolic acids, flavonoids, } \\
\text { and anthocyanins }\end{array}$ & C57BL/6 mice & $\begin{array}{l}20 \% \text { navy bean or } \\
\text { black bean/p.o. }\end{array}$ & 2 weeks & $\begin{array}{l}\text { Anti-oxidant and } \\
\text { anti-inflammatory } \\
\text { activities }\end{array}$ & [120] \\
\hline $\begin{array}{l}\text { Pinto, navy and } \\
\text { black beans }\end{array}$ & $(+)$-catechin & Salmonella typhimurium strains TA98 and TA100 & $\begin{array}{l}2.5,5,10,12.5 \\
15 \text { and } 25 \mu \mathrm{g}\end{array}$ & $24 \mathrm{~h}$ & $\begin{array}{l}\text { Anti-oxidant and } \\
\text { anti-mutagenecity } \\
\text { activities }\end{array}$ & [90] \\
\hline $\begin{array}{l}\text { Pinto, navy and } \\
\text { black beans }\end{array}$ & $\begin{array}{l}\text { Phenolic acids and Lectin-free } \\
\text { fractions }\end{array}$ & Human erythrocytes and Saccharomyces cerevisiae cells & $0.2 \mathrm{mg}$ & $24 \mathrm{~h}$ & $\begin{array}{c}\text { Anti-oxidant and } \\
\text { anti-mutagenic effects }\end{array}$ & [93] \\
\hline $\begin{array}{l}\text { Black and kidney } \\
\text { beans }\end{array}$ & Catechin & In vitro & $50-100 \mu \mathrm{L}$ & $24 \mathrm{~h}$ & $\begin{array}{l}\text { Anti-oxidant and } \\
\text { anti-mutagenic } \\
\text { activities }\end{array}$ & [91] \\
\hline $\begin{array}{l}12 \text { varieties of } \\
\text { non-pigmented } \\
\text { bean, red bean, } \\
\text { speckled bean, } \\
\text { and dark bean }\end{array}$ & $\begin{array}{l}\text { Gallic acid, chlorogenic acid, } \\
\text { epicatechin, myricetin, formononetin, } \\
\text { caffeic acid, and kaempferol }\end{array}$ & $\begin{array}{c}\text { In vitro human epithelial colorectal adenocarcinoma } \\
\text { (Caco-2) cells, breast cancer (MCF-7), and A549 } \\
\text { NSCLC cell line }\end{array}$ & $15-300 \mu \mathrm{L}$ & $36-48 \mathrm{~h}$ & $\begin{array}{l}\text { Anti-oxidant and } \\
\text { anti-proliferative } \\
\text { Activities }\end{array}$ & [121] \\
\hline Black bean & Genistein, non-glycosylated flavonols & $\begin{array}{l}\text { In vitro mammary gland, hepatic and colon cancer } \\
\text { cell lines }\end{array}$ & $50-200 \mu \mathrm{L}$ & $36-48 \mathrm{~h}$ & $\begin{array}{l}\text { Anti-oxidant and } \\
\text { anti-proliferative } \\
\text { activities }\end{array}$ & [122] \\
\hline Black bean & Total phenolics & Wistar albino rats & 200 mg/kg bw/p.o. & 45 days- & $\begin{array}{c}\text { Anti-oxidant } \\
\text { anti-diabetic and } \\
\text { anti-hyperlipidemic } \\
\text { activities }\end{array}$ & [123] \\
\hline Dalia bean & $\begin{array}{l}\text { Coumaric, salicylic, gallic, caffeic acids, } \\
\text { epigallocatechin, rutin and quercetin, } \\
\text { and flavonoids }\end{array}$ & In vitro & $100 \mu \mathrm{L}$ & $24 \mathrm{~h}$ & Anti-oxidant activity & [124] \\
\hline Brazilian bean & $\begin{array}{l}\text { Ferulic, sinapic, chlorogenic, } \\
\text { and hydroxycinnamic acids }\end{array}$ & In vitro & $50 \mu \mathrm{L}$ & $24 \mathrm{~h}$ & Anti-oxidant activity & [125] \\
\hline
\end{tabular}


Table 3. Cont

\begin{tabular}{|c|c|c|c|c|c|c|}
\hline Bean Name & Polyphenols Names & Model/Subjects & Dosage & $\begin{array}{l}\text { Experimental } \\
\text { Period }\end{array}$ & Activities & References \\
\hline $\begin{array}{l}\text { Pinto and black } \\
\text { beans }\end{array}$ & $\begin{array}{l}\text { Total phenolics, phenolic acids, } \\
\text { isoflavones, and anthocyanins }\end{array}$ & In vitro & $50 \mu \mathrm{L}$ & $24 \mathrm{~h}$ & Anti-oxidant activity & {$[64,85,91]$} \\
\hline Brazilian bean & Total phenolics, and phenolic acids & In vitro & $50-100 \mu \mathrm{L}$ & $24 \mathrm{~h}$ & Anti-oxidant activity & [126] \\
\hline Black bean & Total phenolics & In vitro & $200 \mu \mathrm{L}$ & $24 \mathrm{~h}$ & Anti-oxidant activity & {$[127,128]$} \\
\hline Yellow string bean & Total polyphenolics & In vitro & $10-100 \mu \mathrm{L}$ & $24 \mathrm{~h}$ & Anti-oxidant activity & [92] \\
\hline Black bean & Total phenolics & Wistar albino rats & 200 mg/kg bw/p.o. & 45 days & Anti-oxidant activity & [94] \\
\hline Black bean & $\begin{array}{l}(+) \text {-catechin, quercetin, vanillin and } \\
\text { ellagic, caffeic, ferulic, gallic, } \\
\text { chlorogenic, and sinapic acids }\end{array}$ & Human and in vitro & $1 \mathrm{~g} / \mathrm{p} . \mathrm{o}$. & $36-48 \mathrm{~h}$ & $\begin{array}{c}\text { Anti-oxidant activity } \\
\text { and enhance } \\
\text { gastrointestinal } \\
\text { digestion and } \\
\text { simulated colonic } \\
\text { fermentation }\end{array}$ & [129] \\
\hline Dark bean & $\begin{array}{l}p \text {-coumaric, ferulic, sinapic acids, } \\
\text { myricetin, quercetin, kaempferol, } \\
\text { flavanones, hesperetin and } \\
\text { naringenin derivatives }\end{array}$ & $\begin{array}{l}\text { In vitro cell line cultures of Astrocytes (U-373), renal } \\
\text { adenocarcinoma (TK-10), breast adenocarcinoma } \\
\text { (MCF-7) and melanoma (UACC-62) }\end{array}$ & $700 \mu \mathrm{L}$ & $36-48 \mathrm{~h}$ & $\begin{array}{l}\text { Anti-oxidant, } \\
\text { neuroprotective and } \\
\text { anticancer activities }\end{array}$ & {$[61]$} \\
\hline Kidney bean & Total phenolics & Female Sprague Dawley rats & $\begin{array}{c}0,7.5 \%, 15 \%, 30 \% \text { or } \\
60 \% w / w\end{array}$ & 46 days & $\begin{array}{l}\text { Chemoprotective } \\
\text { effect on breast cancer }\end{array}$ & [130] \\
\hline Black bean & Total phenolics & Female Sprague Dawley rats & $\begin{array}{l}7.5 \%, 15 \%, 30 \% \text { or } \\
60 \% w / w\end{array}$ & 46 days & $\begin{array}{l}\text { Chemoprotective } \\
\text { effect on breast cancer }\end{array}$ & [131] \\
\hline $\begin{array}{l}\text { Black, pinto and } \\
\text { kidney beans }\end{array}$ & Tannins & Male Sprague-Dawley rats & $\begin{array}{l}7.5 \%, 15 \%, 30 \% \text { or } \\
\quad 60 \% w / w\end{array}$ & 46 days & $\begin{array}{l}\text { Chemoprotective } \\
\text { effect on breast cancer }\end{array}$ & [132] \\
\hline Black bean & Tannins & Male Sprague-Dawley rats & $2.5 \mathrm{~g} / \mathrm{kg}$ bw $/$ p.o. & 9 weeks & $\begin{array}{l}\text { Chemoprotective } \\
\text { effect on colon cancer }\end{array}$ & [133] \\
\hline $\begin{array}{l}\text { Black and navy } \\
\text { beans }\end{array}$ & Tannins & Male Sprague-Dawley rats & $2.5 \mathrm{~g} / \mathrm{kg}$ bw $/$ p.o. & 9 weeks & $\begin{array}{l}\text { Chemoprotective } \\
\text { effect on colon cancer }\end{array}$ & [134] \\
\hline Black beans & Tannins & Male Sprague-Dawley rats & $2.5 \mathrm{~g} / \mathrm{kg} \mathrm{bw} / \mathrm{p} .0$ & 9 weeks & $\begin{array}{l}\text { Chemoprotective } \\
\text { effect on colon cancer }\end{array}$ & {$[135,136]$} \\
\hline Kidney bean & Tannins & Human HT-29 cell lines & $100 \mu \mathrm{L}$ & $48 \mathrm{~h}$ & $\begin{array}{l}\text { Chemoprotective } \\
\text { effect on colon cancer }\end{array}$ & {$[137]$} \\
\hline Kidney bean & Tannins & $\begin{array}{l}\text { Sprague-Dawley rats and Clostridium butyricum } \\
\text { strain MIYAIRI588 }\end{array}$ & $2.5 \mathrm{~g} / \mathrm{kg}$ bw $/$ p.o. & 9 weeks & $\begin{array}{l}\text { Chemoprotective } \\
\text { effect on colon cancer }\end{array}$ & [138] \\
\hline
\end{tabular}


Table 3. Cont

\begin{tabular}{|c|c|c|c|c|c|c|}
\hline Bean Name & Polyphenols Names & Model/Subjects & Dosage & $\begin{array}{l}\text { Experimental } \\
\text { Period }\end{array}$ & Activities & References \\
\hline Black bean & (+)-catechin & Human HT-29 cell lines & $20 \mathrm{mg}$ & $48 \mathrm{~h}$ & $\begin{array}{c}\text { Chemoprotective } \\
\text { effect on colon cancer }\end{array}$ & [139] \\
\hline Black bean & Flavonoids & In vitro & $5 \mathrm{mg}$ & $24 \mathrm{~h}$ & $\begin{array}{c}\text { Cholesterol-lowering } \\
\text { effects }\end{array}$ & [140] \\
\hline Black bean & $\begin{array}{c}\text { Orientin, isoorientin, rutin } \\
\text { myricetin-3-O-rhamnoside, } \\
\text { isorhamnetin-3-O-glucoside, } \\
\text { isoquercitrin, myricetin, luteolin, } \\
\text { quercetin, kaempferol, hyperoside, } \\
\text { luteolin-7-O-glucoside, } \\
\text { kaempferol-glucuronide, caffeine, } \\
\text { isorhamnetin-3-O-glucoside, } \\
\text { hydroxycinnamic acid, } \\
\text { and proanthocyanidins }\end{array}$ & Male Wistar rats & $\begin{array}{c}2 \% \text { bean seed coat } \\
\text { extract/p.o. }\end{array}$ & 7 days & $\begin{array}{l}\text { Hypoglycemic and } \\
\text { hypolipidemic effects }\end{array}$ & [141] \\
\hline Black bean & Total phenolics & Wistar albino rats & 200 mg/kg bw/p.o. & 45 days & $\begin{array}{c}\text { Hypoglycemic and } \\
\text { hypolipidemic effects }\end{array}$ & [142] \\
\hline Black bean & Flavonoids & Male Wistar rats & $200,400 \mathrm{mg} / \mathrm{kg}$ bw & 7 days & Hypoglycemic effect & {$[143,144]$} \\
\hline $\begin{array}{l}\text { Navy and pinto } \\
\text { beans }\end{array}$ & Flavonoids & Male Wistar rats & $\begin{array}{c}50,200,500 \mathrm{mg} / \mathrm{kg} \\
\text { bw }\end{array}$ & 15 days & $\begin{array}{l}\text { Hypoglycemic effect } \\
\text { and anti-obesity effect }\end{array}$ & [145] \\
\hline Kidney bean & Flavonoids & Male Wistar rats & $\begin{array}{c}50,200,500 \mathrm{mg} / \mathrm{kg} \\
\text { bw }\end{array}$ & 21 days & $\begin{array}{l}\text { Hypoglycemic effect } \\
\text { and anti-obesity effect }\end{array}$ & [146] \\
\hline Black bean & Flavonoids & Obese Zucker fa/fa rats & 50 and $500 \mathrm{mg} / \mathrm{kg} \mathrm{bw}$ & $\begin{array}{l}3-7 \text { days, } \\
20 \text { days }\end{array}$ & $\begin{array}{l}\text { Hypoglycemic effect } \\
\text { and anti-obesity effect }\end{array}$ & [147] \\
\hline Black bean & Catechin & Male Wistar rats & $50 \mathrm{mg} / \mathrm{kg}$ bw & 21 days & $\begin{array}{l}\text { Hypoglycemic effect } \\
\text { and anti-obesity effect }\end{array}$ & [109] \\
\hline Black bean & Flavonoids & CD1 mice & $200,400 \mathrm{mg} / \mathrm{kg}$ bw & 45 days & $\begin{array}{l}\text { Hypoglycemic effect } \\
\text { and anti-obesity effect }\end{array}$ & [148] \\
\hline Black bean & $\begin{array}{l}\text { Total phenolic, tannins and } \\
\text { anthocyanins }\end{array}$ & CD1 mice & $200,400 \mathrm{mg} / \mathrm{kg}$ bw & 21 days & $\begin{array}{l}\text { Hypoglycemic effect } \\
\text { and anti-obesity effect }\end{array}$ & [149] \\
\hline Black bean & Quercetin 3-O-glucoside & Rat hepatocytes and C57BL/6 mice & $25 \mathrm{mg}$ & $48 \mathrm{~h}$ & $\begin{array}{l}\text { Hypolipidemic } \\
\text { activity }\end{array}$ & [140] \\
\hline
\end{tabular}

Expansion: b.w- body weight; $w / w$ - weight/weight; p.o.- per oral. 


\subsection{Anti-Diabetic Activity}

Venn and Mann [150] have strongly suggested that the regular consumption of dry common beans is beneficial in the prevention and management of diabetes. Clinical studies show that consumption of three or more servings of beans in a week decreases the menace of diabetes almost by $35 \%$, as compared to less or non-consumption of beans [151]. In vitro anti-diabetic studies of common beans have showed a greater inhibition of $\alpha$-amylase, $\alpha$-glucosidase and dipeptidyl peptidase-IV, which have found to be anti-hyperglycemic activities due to their phenolic compounds such as flavonoids and their glucosides of delphinidin, petunidin, and malvidin, anthocyanins, catechin, myricetin 3-O-arabinoside, epicatechin, vanillic acid, syringic acid, and $O$-coumaric acid [23,101,152,153]. In vivo studies have also demonstrated that beans containing phenolic compounds reduce blood glucose, glycosylated hemoglobin and elevated insulin levels in the animals [104-106,131]. Similarly, Roman-Ramos et al. [154] have demonstrated that anti-hyperglycemic effect of kidney beans in healthy rats revealed $21 \%$ reduction in the graph plot under glucose tolerance curve when compared with $16 \%$ of the standard diabetic drug. Epidemiological studies associated with Chinese population have shown that regular intakes of common beans are inversely connected with the risk of type-2 diabetes [155]. Gupta et al. [103] and Tang et al. [156] have also studied in 56 diabetic subjects based on traditional and Ayurvedic principles, which have revealed that the regular consumption of black bean for three months reduces plasma glucose and glycosylated hemoglobin. The results showed that black bean ameliorates type- 2 diabetes, which is due to black beans containing total phenolic, tannins and anthocyanins. In vitro and in vivo studies of common beans exerting anti-diabetic activities are summarized in Table 3.

\subsection{Anti-Obesity and Cardioprotective Activity}

Metabolic syndrome is the set of metabolic conditions connected with the threat of cardiovascular diseases, increased triglycerides (TG), total cholesterol (TC), low density lipoprotein (LDL), very low density lipoprotein (VLDL), blood pressure (BP), and glucose as well as lower levels of HDL and central adiposity $[157,158]$. Regular intake of dry common beans has proven to be favorable for healthy subjects as well as obese individuals by decreasing serum TC and LDL and elevating HDL [159]. Epidemiological and clinical studies have demonstrated that consumption of common beans inversely connected with the risk of cardiovascular and coronary arterial diseases $[158,160]$. Further, these studies revealed that consumption of beans four or more times per week reduced the risks of coronary arterial diseases $(22 \%)$ and cardiovascular diseases $(11 \%)$ : serum TC declines of about $1 \%$ decrease the risk of coronary heart disease by $2 \%$, while serum LDL declines of about $1 \%$ reduce the risk of both diseases by about $1 \%$ [160]. Two weeks of regular consumption of baked beans by hypercholesterolemic individuals showed a significant reduction of TC $(12 \%)$ and LDL (15\%) [161]. Another clinical trial has also investigated on the hypercholesterolemic subjects with consumption of $275 \mathrm{~g}$ of navy beans for three weeks found a reduction of both serum TC, and LDL up to $24 \%$ [162]. Eight weeks of consumption of one cup serving baked beans by hypercholesterolemic individuals showed a marked reduction of TC (6\%) and LDL (5\%) [160]. Similarly, consumption of dried, cooked pinto beans (130 g) four times a week significantly decrease serum TC and LDL in healthy individuals, resulted in decreasing the risk of cardiovascular disease by $20 \%$ [158]. Azuki bean juice supplementation to young women also showed significantly decrease TC and TG levels and proved to be anti-hypercholesterolemic [163]. Similarly, consumption of one serving of cooked beans on regular basis inversely associated with the risk of myocardial infarction up to $38 \%$ [164].

In vivo anti-obesity and cardioprotective studies of common beans have showed a greater reduction of TC, TG, free fatty acids (FFA), phospholipids, and FA composition of total lipids, and was have found to have anti-hyperlipidemic activities due to their phenolic compounds such as quercetin, quercetin 3-O-glucoside, kaempferol, $p$-coumaric acid, ferulic acid, $p$-hydroxybenzoic acid, vanillic acid [116,165], orientin, isoorientin, rutin, myricetin-3-rhamnoside, hyperoside, isorhamnetin-3-O-glucoside, isoquercitrin, myricetin, luteolin, quercetin, luteolin-7-O-glucoside, 
kaempferol-glucuronide, kaempferol, isorhamnetin-3-O-glucoside, caffeine, hydroxycinnamic acid, and proanthocyanidins [141]. In vitro and in vivo studies of common beans exerting anti-obesity and cardiovascular activities are summarized in Table 3.

\subsection{Anti-Mutagenic and Anti-Carcinogenic Activities}

Normally, the generation of ROS and oxidative stress damage macromolecules such as lipid, protein RNA, and DNA, which may cause chronic degenerative diseases, including cancer [166,167]. However, the occurrence of cancer can be decreased by lifestyle and dietary habit changes. Studies have also suggested that diets rich in common beans reduce the greater risk of various cancers including colon, breast, and prostate $[130,134,137]$. A larger study conducted in 41 countries found that the consumption of common beans reduced the morbidity by cancers such as colon, breast, and prostate [168]. Further, studies have revealed that consumption of beans two or more times per week reduced the risks of colon cancer up to $47 \%$ [107], prostate cancer about 22\% [169] and breast cancer about $67 \%$ [170]. In vivo studies have also suggested consumption of beans reduced risk of various cancers [132-136]. Hangen and Bennink [171] investigated diets fed with black beans in rats and observed the lower the incidence of total tumor (54\%) and adenocarcinoma (75\%). Similarly, the effect of navy beans decreased the occurrence of total tumor (59\%) and adenocarcinoma (44\%). The anti-carcinogenic and anti-mutagenic activities of beans are highly associated with the presence of phenolic compounds as well as other bioactive compounds [62,93]. Phenolic compounds have the potential to inhibit mutagenic agents including polycyclic aromatic hydrocarbons, nitrosamines, and mycotoxins by inhibiting activation enzymes, provoking detoxification enzymes and intonation of commencement of mutagens [172,173]. Furthermore, common beans possess anti-carcinogenic and anti-mutagenic properties due to their phenolic compounds interacting with ultimate toxicants or mutagens, scavenging activities of phenolics, and inhibition of metabolism of the ultimate mutagen $[62,76,174]$. In vitro and in vivo studies on chemopreventive and anti-mutagenic activities of common beans are summarized in Table 3 .

\subsection{Anti-Inflammatory Activity}

Common beans contain phenolic compounds (phenolic acids, flavonoids, and anthocyanins) and non-digestible fermentable components (short-chain fatty acid precursors) with demonstrated anti-oxidant and anti-inflammatory activities. Experimental studies associated with modulation of inflammatory-related cell signaling pathways by common beans have been well established. In an animal study, C57BL / 6 mice fed a 20\% navy bean or black bean flour-containing diet showed significantly reduced dextran sodium sulfate induced experimental colitis and inflammation-related parameters (IL-1 $\beta$, TNF $\alpha$, IFN $\gamma$, IL-17A, and IL-9), increased histological injury score and apoptosis, and alleviated symptoms of colitis and colon inflammation [15]. Common beans possess various bioactive compounds including flavonoids and anthocyanins, which significantly reduced the activity of murine macrophages through the inhibition of pro-inflammatory gene expression without cytotoxicity [117-119]. Similarly, human-randomized, controlled, crossover trials have also demonstrated that three-day intake of $100 \mathrm{~g}$ of black bean meal and soup improved the arthritic condition by significantly reducing pain and inflammation [119]. The immunomodulatory effects of $20 \%$ navy bean or black bean or cranberry bean administration in C57BL/ 6 mice for two weeks showed a significant reduction in colonic mucosal damage and inflammation in response to dextran sodium sulfate. The results further demonstrated that common bean containing bioactive compounds including phenolic acids, flavonoids, and anthocyanins provoke prominent immune response [26,120]. In vitro and in vivo studies of common beans exerting anti-inflammatory activities are summarized in Table 3. 


\section{Conclusions}

Dry common beans are consumed in diets worldwide, and play a significant function in human nutrition, especially as a source rich in proteins, carbohydrates, dietary fibers, vitamins, minerals, phytochemicals and other micronutrients, as well as low saturated fat content. Besides these nutrients, common beans possess enormous quantities of polyphenols and other metabolites, have anti-oxidant activities, major role in health-promoting effects, and protect against various diseases including diabetes, CVD, cancer, and microbial infections. These health benefits of beans are generally acquired from direct attributes, including their high content of nutrients, as well as replacement in the diet, when they substitute for animal products. It can therefore be concluded that health-promoting effects are directly proportional to the increase in bean intake. In addition, long-term clinical studies are urgently needed to warrant the therapeutic benefit of polyphenols rich common beans. In addition, the synergistic effects of polyphenol-rich common bean with other bioactive compounds on biological functions would be a recommendation for further studies. This investigation proves the efficacy of bioactive compounds in common bean, and enhances the therapeutic options for various diseases.

Acknowledgments: The work was jointly supported by two grants R201627 and R201714 from Beijing Normal University-Hong Kong Baptist University United International College, Zhuhai, Guangdong, China.

Author Contributions: Kumar Ganesan and Baojun Xu conceived and designed the review; Kumar Ganesan wrote the paper; and Baojun Xu critically revised and improved the manuscript.

Conflicts of Interest: The authors declared that no conflicts of interest.

\section{Abbreviations}

$\begin{array}{ll}\text { b.w. } & \text { Body weight } \\ \text { BP } & \text { Blood pressure } \\ \text { CAT } & \text { Catalase } \\ \text { DPPH } & \text { 2,2'-Diphenyl-1-picrylhydrazyl } \\ \text { FA } & \text { Fatty acids } \\ \text { FFA } & \text { Free fatty acids } \\ \text { GR } & \text { Glutathione reductase } \\ \text { GSH } & \text { Reduced glutathione } \\ \text { GST } & \text { Glutathione-s-transferase } \\ \text { HDL } & \text { High density lipoprotein } \\ \text { IL } & \text { Interleukin } \\ \text { i.p. } & \text { Intraperitoneal } \\ \text { i.v. } & \text { Intravenous } \\ \text { kDa } & \text { Kilo daltons } \\ \text { LDL } & \text { Low density lipoprotein } \\ \text { MTT } & \text { 3-(4,5-Dimethylthiazol-2-Yl)-2,5-diphenyltetrazolium bromide } \\ \text { p.o. } & \text { Per oral } \\ \text { ROS } & \text { Reactive oxygen species } \\ \text { SOD } & \text { Super oxide dismutase } \\ \text { TBARS } & \text { Thiobarbituric acid reactive substances } \\ \text { TC } & \text { Total cholesterol } \\ \text { TG } & \text { Triglycerides } \\ \text { TNF- } \alpha & \text { Tumour necrosis factor } \alpha \\ \text { VLDL } & \text { Very low density lipoprotein } \\ w / w & \text { Weight/weight } \\ & \end{array}$

\section{References}

1. Sharma, A.; Kaur, M.; Katnoria, J.K.; Nagpal, A.K. Polyphenols in food: Cancer prevention and apoptosis induction. Curr. Med. Chem. 2017. [CrossRef] [PubMed] 
2. Ganesan, K.; Xu, B. A critical review on polyphenols and health benefits of black soybeans. Nutrients $2017,9$. [CrossRef]

3. McDougall, G.J. Phenolic-enriched foods: Sources and processing for enhanced health benefits. Proc. Nutr. Soc. 2017, 76, 163-171. [CrossRef] [PubMed]

4. Aparicio-Fernández, X.; García-Gasca, T.; Yousef, G.G.; Lila, M.A.; González de Mejía, E.; Loarca-Piña, G. Chemopreventive activity of polyphenolics from black Jamapa Bean (Phaseolus vulgaris L.) on HeLa and HaCaT cells. J. Agric. Food Chem. 2006, 54, 2116-2122. [CrossRef] [PubMed]

5. Aparicio-Fernández, X.; Yousef, G.G.; Loarca-Piña, G.; González de Mejía, E.; Lila, M.A. Characterization of polyphenolics in the seed coat of Black Jamapa bean (Phaseolus vulgaris L.). J. Agric. Food Chem. 2005, 53, 4615-4622. [CrossRef] [PubMed]

6. Aparicio-Fernández, X.; Manzo-Bonilla, L.; Loarca-Piña, G. Comparison of antimutagenic activity of phenolic compounds in newly harvested and stored common beans Phaseolus Vulgaris against aflatoxin B1. J. Food Sci. 2005, 70, S73-S78. [CrossRef]

7. Beninger, C.W.; Hosfield, G.L. Antioxidant activity of extracts, condensed tannin fractions, and pure flavonoids from Phaseolus vulgaris L. seed coat color genotypes. J. Agric. Food Chem. 2003, 51, 7879-7883. [CrossRef] [PubMed]

8. Cardador-Martínez, A.; Loarca-Piña, G.; Oomah, B.D. Antioxidant activity in common beans (Phaseolus vulgaris L.). J. Agric. Food Chem. 2002, 50, 6975-6980. [CrossRef] [PubMed]

9. Guzmán-Maldonado, S.H.; Paredes-López, O. Functional products of plants indigenous of Latin America: Amaranth, quinoa, common beans and botanicals. In Functional Foods. Bichemical and Processing Aspects; Mazza, G., Ed.; Thechnomic: Lancaster, PA, USA, 1998; pp. 39-328.

10. Hangen, L.; Bennink, M.R. Consumption of Black Beans and Navy Beans (Phaseolus vulgaris) Reduced azoxymethane-induced colon cancer in rats. Nutr. Cancer 2002, 44, 60-65. [PubMed]

11. Queiroz-Monici, K.S.; Costa, G.E.A.; da Silva, N.; Reis, S.M.P.M.; de Oliveira, A.C. Bifidogenic effect of dietary fiber and resistant starch from leguminous on the intestinal microbiota of rats. Nutrition 2005, 21, 602-609. [CrossRef] [PubMed]

12. FAO. Estadísticas de Fríjol Seco. Available online: http://www.fao.org/faostat/en/\#data/QC (accessed on 26 May 2014).

13. Adams, M.W. Energy inputs in dry bean production. In Handbook of Energy Utilization in Agriculture; Pimentel, D., Ed.; CRC Press: Boca Raton, FL, USA, 1980; pp. 123-126.

14. Mitchell, D.C.; Lawrence, F.R.; Hartman, T.J.; Curran, J.M. Consumption of dry beans, peas, and lentils could improve diet quality in the US population. J. Am. Diet. Assoc. 2009, 109, 909-913. [CrossRef] [PubMed]

15. Zhang, C.; Monk, J.M.; Lu, J.T.; Zarepoor, L.; Wu, W.; Liu, R.; Pauls, K.P.; Wood, G.A.; Robinson, L.; Tsao, R.; et al. Cooked navy and black bean diets improve biomarkers of colon health and reduce inflammation during colitis. Br. J. Nutr. 2014, 111, 1549-1563. [CrossRef] [PubMed]

16. Borresen, E.C.; Brown, D.G.; Harbison, G.; Taylor, L.; Fairbanks, A.; O’Malia, J.; Bazan, M.; Rao, S.; Bailey, S.M.; Wdowik, M.; et al. A randomized controlled trial to increase navy bean or rice bran consumption in colorectal cancer survivors. Nutr. Cancer 2016, 68, 1269-1280. [CrossRef] [PubMed]

17. Borresen, E.C.; Jenkins-Puccetti, N.; Schmitz, K.; Brown, D.G.; Pollack, A.; Fairbanks, A.; Wdowik, M.; Rao, S.; Nelson, T.L.; Luckasen, G.; et al. A pilot randomized controlled clinical trial to assess tolerance and efficacy of navy bean and rice bran supplementation for lowering cholesterol in children. Glob. Pediatr. Health 2017, 4. [CrossRef] [PubMed]

18. Lestari, L.A.; Huriyati, E.; Marsono, Y. The development of low glycemic index cookie bars from foxtail millet (Setaria italica), arrowroot (Maranta arundinacea) flour, and kidney beans (Phaseolus vulgaris). J. Food Sci. Technol. 2017, 54, 1406-1413. [CrossRef] [PubMed]

19. Monk, J.M.; Zhang, C.P.; Wu, W.; Zarepoor, L.; Lu, J.T.; Liu, R.; Pauls, K.P.; Wood, G.A.; Tsao, R.; Robinson, L.E.; et al. White, and dark kidney beans reduce colonic mucosal damage and inflammation in response to dextran sodium sulfate. J. Nutr. Biochem. 2015, 26, 752-760. [CrossRef] [PubMed]

20. Chao, W.W.; Chung, Y.C.; Shih, I.P.; Wang, H.Y.; Chou, S.T.; Hsu, C.K. Red bean extract inhibits lipopolysaccharide-induced inflammation and $\mathrm{H}_{2} \mathrm{O}_{2}$-induced oxidative stress in RAW 264.7 macrophages. J. Med. Food. 2015, 18, 724-730. [CrossRef] [PubMed]

21. Chan, Y.S.; $\mathrm{Ng}$, T.B. Northeast red beans produce a thermostable and $\mathrm{pH}$-stable defensin-like peptide with potent antifungal activity. Cell Biochem. Biophys. 2013, 66, 637-648. [CrossRef] [PubMed] 
22. Chan, Y.S.; Wong, J.H.; Fang, E.F.; Pan, W.; Ng, T.B. A hemagglutinin from northeast red beans with immunomodulatory activity and anti-proliferative and apoptosis-inducing activities toward tumor cells. Protein Pept. Lett. 2013, 20, 1159-1169. [CrossRef] [PubMed]

23. Mojica, L.; Berhow, M.; Gonzalez de Mejia, E. Black bean anthocyanin-rich extracts as food colorants: Physicochemical stability and antidiabetes potential. Food Chem. 2017, 229, 628-639. [CrossRef] [PubMed]

24. Yin, C.; Wong, J.H.; Ng, T.B. Isolation of a hemagglutinin with potent antiproliferative activity and a large antifungal defensin from Phaseolus vulgaris cv. Hokkaido Large Pinto Beans. J. Agric. Food Chem. 2015, 63, 5439-5448. [CrossRef] [PubMed]

25. Ojeda, A.G.; Wrobel, K.; Escobosa, A.R.; Elguera, J.C.; Garay-Sevilla, M.E.; Wrobel, K. Molybdenum and copper in four varieties of common bean (Phaseolus vulgaris): New data of potential utility in designing healthy diet for diabetic patients. Biol. Trace Elem. Res. 2015, 163, 244-254. [CrossRef] [PubMed]

26. Monk, J.M.; Lepp, D.; Zhang, C.P.; Wu, W.; Zarepoor, L.; Lu, J.T.; Pauls, K.P.; Tsao, R.; Wood, G.A.; Robinson, L.E.; et al. Diets enriched with cranberry beans alter the microbiota and mitigate colitis severity and associated inflammation. J. Nutr. Biochem. 2016, 28, 129-139. [CrossRef] [PubMed]

27. Haddad, E.H.; Tanzman, J.S. What do vegetarians in the United States eat? Am. J. Clin. Nutr. 2003, 78, 626-632.

28. Messina, V. Nutritional and health benefits of dried beans. Am. J. Clin. Nutr. 2014, 100, 437-442. [CrossRef] [PubMed]

29. McCrory, M.A.; Hamaker, B.R.; Lovejoy, J.C.; Eichelsdoerfer, P.E. Pulse consumption, satiety, and weight management. Adv. Nutr. 2010, 1, 17-30. [CrossRef] [PubMed]

30. Darmadi-Blackberry, I.; Wahlqvist, M.L.; Kouris-Blazos, A.; Steen, B.; Lukito, W.; Horie, Y.; Horie, K. Legumes: The most important dietary predictor of survival in older people of different ethnicities. Asia Pac. J. Clin. Nutr. 2004, 13, 217-220. [PubMed]

31. Chávez-Mendoza, C.; Sánchez, E. Bioactive compounds from Mexican varieties of the common bean (Phaseolus vulgaris): Implications for health. Molecules 2017, 22, 1360. [CrossRef] [PubMed]

32. Suárez-Martínez, S.E.; Ferriz-Martínez, R.A.; Campos-Vega, R.; Elton-Puente, J.E.; de la Torre Carbot, K.; García-Gasca, T. Bean seeds: Leading nutraceutical source for human health. CyTA J. Food 2016, 14, $131-137$. [CrossRef]

33. Ulloa, J.A.; Rosas, U.P.; Ramírez, R.J.C.; Rangel, U.B.E. El frijol (Phaseolus vulgaris): Su importancia nutricionaly como fuente de fitoquímicos. [Beans (Phaseolus vulgaris): Their nutritional importance and source of phytochemicals]. Rev. Fuente 2011, 3, 5-9.

34. Mederos, Y. Indicadores de la calidad en el grano de frijol (Phaseolus vulgaris L.). [Quality indicators in bean (Phaseolus vulgaris L.)]. Cultiv. Trop. 2006, 27, 55-63.

35. Díaz-Batalla, L.; Widholm, J.M.; Fahey, G.C.; Castaño-Tostado, E.; Paredes-López, O. Chemical components with health implications in wild and cultivated Mexican common bean seeds (Phaseolus vulgaris L.). J. Agric. Food Chem. 2006, 54, 2045-2052. [CrossRef] [PubMed]

36. Machado, C.M.; Ferruzzi, M.G.; Nielsen, S.S. Impact of the hard-to-cook phenomenon on phenolic antioxidants in dry beans (Phaseolus vulgaris). J. Agric. Food Chem. 2008, 56, 3102-3110. [CrossRef] [PubMed]

37. Aguilera, Y.; Estrella, I.; Benitez, V.; Esteban, R.M.; Martin-Cabrejas, M.A. Bioactive phenolic compounds and functional properties of dehydrated beans flours. Food Res. Int. 2010, 44, 774-780. [CrossRef]

38. López-Amorós, M.L.; Hernández, T.; Estrella, I. Effect of germination on legume phenolic compounds and their antioxidant activity. J. Food Compos. Anal. 2006, 19, 277-283. [CrossRef]

39. Butt, M.S.; Batool, R. Nutritional and functional properties of some promising legume protein isolates. Pak. J. Nutr. 2010, 9, 373-379. [CrossRef]

40. Genovese, M.I.; Lajolo, F.M. Atividade inibito ria de tripsina do feijao (Phaseolus vulgaris L.): Avaliacao. critica dos metodos de determinacao. Arch. Latinoam. Nutr. 2001, 51, 386-394. [PubMed]

41. Costa, G.E.A.; Queiroz-Monici, K.S.; Reis, S.M.P.M.; Oliveira, A.C. Chemical composition, dietary fibre and resistant starch contents of raw and cooked pea, common bean, chickpea and lentil legumes. Food Chem. 2006, 94, 327-330. [CrossRef]

42. Tang, C.H.; Sun, X. Structure-physicochemical function relationships of 7 S globulins (vicilins) from red bean (Phaseolus anglaris) with different polypeptide constituents. Food Hydrocoll. 2011, 25, 536-544. [CrossRef]

43. Tang, C.H.; Sun, X. A comparative study of physicochemical and conformational properties in three vicilins from Phaseolus legumes: Implications for the structure-function relationship. Food Hydrocoll. 2011, 25, 315-324. [CrossRef] 
44. Tang, C.H.; Sun, X.; Yin, S.W. Physicochemical, functional and structural properties of vicilin-rich protein isolate from three Phaseolus legumes: Effect of heat treatment. Food Hydrocoll. 2009, 23, 1771-1778. [CrossRef]

45. Adebowale, Y.A.; Adeyemi, I.A.; Oshodi, A.A.; Niranjan, K. Isolation, fractionation and characterization of proteins from Mucuna bean. Food Chem. 2007, 104, 287-299. [CrossRef]

46. Seena, S.; Sridhar, K.R.; Bajia, B. Biochemical and biological evaluation of ANF unconventional legume, Canavalia maritima of coastal sand dunes of India. Trop. Subtrop. Agroecosyst. 2005, 5, 1-14.

47. Slupski, J. Effect of cooking and sterilization on the composition of amino acids on immature seeds of flageolet beans (Phaseolus vulgaris L.) cultivars. Food Chem. 2010, 121, 1171-1176. [CrossRef]

48. Welch, R.M.; House, W.A.; Beebe, S.; Cheng, Z. Genetic selection for enhanced bioavailable levels of iron in bean (Phaseolus vulgaris L.) seeds. J. Agric. Food Chem. 2000, 48, 3576-3580. [CrossRef] [PubMed]

49. Shimelis, E.A.; Rakshit, S.K. Proximate composition and physico-chemical properties of improved dry bean (Phaseolus vulgaris L.) varieties grown in Ethiopia. LWT 2005, 38, 331-338. [CrossRef]

50. Champ, M.M. Non-nutrient bioactive substances of pulses. Br. J. Nutr. 2002, 88, 307-319. [CrossRef] [PubMed]

51. Muzquiz, M.; Burbano, C.; Ayet, G.; Pedrosa, M.M.; Cuadrado, C. The investigation of antinutritional factors in Phaseolus vulgaris. Environmental and varietal differences. Biotechnol. Agron. Soc. Environ. 1999, 3, 210-216.

52. Ricroft, C.E.; Jones, M.R.; Gibson, G.R.; Rastall, R.A. A comparative in vitro evaluation of the fermentation properties of prebiotic oligosaccharides. J. Appl. Microbiol. 2001, 91, 878-887. [CrossRef]

53. Midorikawa, K.; Murata, M.; Oikawa, S.; Hiraku, Y.; Kawanishi, S. Protective effect of phytic acid on oxidative DNA damage with reference to cancer chemoprevention. Biochem. Biophys. Res. Commun. 2001, 288, 552-557. [CrossRef] [PubMed]

54. Mathers, J.C. Pulses and carcinogenesis: Potential for prevention of colon, breast and other cancers. Br. J. Nutr. 2002, 88, 272-279. [CrossRef] [PubMed]

55. Lajolo, F.M.; Genovese, M.I. Nutritional significance of lectin and enzyme inhibitors from legumes. J. Agric. Food Chem. 2002, 50, 6592-6598. [CrossRef] [PubMed]

56. Phillippy, B.Q. Inositol phosphates in food. Adv. Food Nutr. Res. 2003, 45, 1-60. [PubMed]

57. Iqbal, A.; Khalil, I.A.; Ateeq, N.; Khan, M.S. Nutritional quality of important food legumes. Food Chem. 2006, 97, 331-335. [CrossRef]

58. United States Department of Agriculture (USDA). Agricultural Research Service, National Nutrient Database for Standard Reference Release 28. Nutrient Database Laboratory Homepage. Available online: https: / / ndb.nal.usda.gov/ndb/search/list (accessed on 14 July 2016).

59. Golam Masum Akond, A.S.M.; Khandaker, L.; Berthold, J.; Gates, J.; Peters, K.; Delong, H.; Hossain, K. Anthocyanin, total polyphenols and antioxidant activity of common bean. Am. J. Food Technol. 2011, 6, 385-394.

60. Ren, S.C.; Liu, Z.L.; Wang, P. Proximate composition and flavonoids content and in vitro antioxidant activity of 10 varieties of legume seeds grown in China. J. Med. Plants Res. 2012, 6, 301-308.

61. López, A.; El-Naggar, T.; Dueñas, M.; Ortega, T.; Estrella, I.; Hernández, T.; Gómez-Serranillos, M.P.; Palomino, O.M.; Carretero, M.E. Effect of cooking and germination on phenolic composition and biological properties of dark beans (Phaseolus vulgaris L.). Food Chem. 2013, 138, 547-555. [CrossRef] [PubMed]

62. Cardador-Martinez, A.; Castano-Tostado, E.; Loarca-Pina, G. Antimutagenic activity of natural phenolic compounds present in the common bean (Phaseolus vulgaris) against aflatoxin B1. Food Addit. Contam. 2002, 19, 62-69. [CrossRef] [PubMed]

63. Beninger, C.W.; Gu, L.; Prior, R.L.; Junk, D.C.; Vandenberg, A.; Bett, K.E. Changes in polyphenols of the seed coat during the after-darkening process in pinto beans (Phaseolus vulgaris L.). J. Agric. Food Chem. 2005, 53, 7777-7782. [CrossRef] [PubMed]

64. $\mathrm{Xu}$, B.J.; Chang, S.K. Total phenolic content and antioxidant properties of Eclipse black beans (Phaseolus vulgaris L.) as affected by processing methods. J. Food Sci. 2008, 73, 19-27. [CrossRef] [PubMed]

65. Akillioglu, H.G.; Karakaya, S. Changes in total phenols, total flavonoids, and antioxidant activities of common beans and pinto beans after soaking, cooking, and in vitro digestion process. Food Sci. Biotechnol. 2010, 19, 633-639. [CrossRef]

66. de Lima, P.F.; Colombo, C.A.; Chiorato, A.F.; Yamaguchi, L.F.; Kato, M.J.; Carbonell, S.A. Occurrence of isoflavonoids in Brazilian common bean germplasm (Phaseolus vulgaris L.). J. Agric. Food Chem. 2014, 62, 9699-9704. [CrossRef] [PubMed] 
67. Ranilla, L.G.; Genovese, M.I.; Lajolo, F.M. Polyphenols and antioxidant capacity of seed coat and cotyledon from Brazilian and Peruvian bean cultivars (Phaseolus vulgaris L.). J. Agric. Food Chem. 2007, 55, 90-98. [CrossRef] [PubMed]

68. Ávalos, G.A.; Pérez-Urria, C.E. Metabolismo secundario de plantas. [Secondary plant metabolism]. Reduca (Biología). Ser. Fisiol. Veg. 2009, 2, 119-145.

69. Reynoso Camacho, R.; del Carmen Ríos Ugalde, M.; Torres Pacheco, I.; Acosta Gallegos, J.A.; Palomino Salinas, A.C.; Ramos Gómez, M.; González Jasso, E.; Horacio Guzmán, Y.S.H. Common bean (Phaseolus vulgaris L.) consumption and its effects on colon cancer in Sprague-Dawley rats. Agric. Téc. Méx. 2007, 33, 43-52.

70. Juárez-López, B.A.; Aparicio-Fernández, X. Polyphenolics concentration and antiradical capacity of common bean varieties (Phaseolus vulgaris L.) after thermal treatment. In Food Science and Food Biotechnology Essentials: A Contemporary Perspective, 1st ed.; Nevárez-Moorillón, G.V., Ortega-Rivas, E., Eds.; Asociación Mexicana de Ciencia de los Alimentos, A.C [Mexican Association of Food Science]: Durango, Mexico, 2012; pp. $25-33$.

71. Lin, L.Z.; Harnly, J.M.; Pastor-Corrales, M.S.; Luthria, D.L. The polyphenolic profiles of common beans (Phaseolus vulgaris L.). Food Chem. 2008, 107, 399-410. [CrossRef] [PubMed]

72. Hayat, I.; Ahmad, A.; Masud, T.; Ahmed, A.; Bashir, S. Nutritional and health perspectives of beans (Phaseolus vulgaris L.): An overview. Crit. Rev. Food Sci. Nutr. 2014, 54, 580-592. [CrossRef] [PubMed]

73. Ariga, T.; Hamano, M. Radical scavenging action and its mode in procyanidins B-1 and B-3 from adzuki beans to peroxyl radicals. Agric. Biol. Chem. 1990, 54, 2499-2504.

74. Tsuda, T.; Ohshima, K.; Kawakishi, S.; Osawa, T. Antioxidative pigments isolated from the seeds of Phaseolus vulgaris L. J. Agric. Food Chem. 1994, 42, 248-251. [CrossRef]

75. Guzman-Maldonado, G.H.; Castellanos, J.; De Mej1a, E.G. Relationship between theoretical and experimentally detected tannin content of common bean Phaseolus vulgaris L. Food Chem. 1996, 55, 333-335. [CrossRef]

76. De Mej1a, E.G.; Castano-Tostado, E.; Loarca-Pina, G. Antimutagenic effects of natural phenolic compounds in beans. Mutat. Res. 1999, 441, 1-9. [CrossRef]

77. Takeoka, G.R.; Dao, L.T.; Full, G.H.; Wong, R.Y.; Harden, L.A.; Edwards, R.H.; Berrios, S. Characterization of black bean (Phaseolus vulgaris L.) anthocyanins. J. Agric. Food Chem. 1997, 45, 3395-3400. [CrossRef]

78. Espinosa-Alonso, L.G.; Lygin, A.; Widholm, J.M.; Valverde, M.E.; Paredes-Lopez, O. Polyphenols in wild and weedy Mexican common beans (Phaseolus vulgaris L.). J. Agric. Food Chem. 2006, 54, 4436-4444. [CrossRef] [PubMed]

79. Jun, S.; Shin, S.; Joung, H. Estimation of dietary flavonoid intake and major food sources of Korean adults. Br. J. Nutr. 2016, 115, 480-489. [CrossRef] [PubMed]

80. Guajardo-Flores, D.; García-Patiño, M.; Serna-Guerrero, D.; Gutiérrez-Uribe, J.A.; Serna-Saldívar, S.O. Characterization and quantification of saponins and flavonoids in sprouts, seed coats, and cotyledons of germinated black beans. Food Chem. 2012, 134, 1312-1319. [CrossRef] [PubMed]

81. Choung, M.G.; Choi, B.R.; An, Y.N.; Chu, Y.H.; Cho, Y.S. Anthocyanin profile of Korean cultivated kidney bean (Phaseolus vulgaris L.). J. Agric. Food Chem. 2003, 51, 7040-7043. [CrossRef] [PubMed]

82. Lima, J.E.; Sampaio, A.L.F.; Henriques, M.M.O.; Barja-Fidalgo, C. Lymphocyte activation and cytokine production by Pisum sativum agglutinin (PSA) in vivo and in vitro. Immunopharmacology 1999, 41, 147-155. [CrossRef]

83. Huber, K.; Brigide, P.; Bretas, E.B.; Canniatti-Brazaca, S.G. Phenolic acid, flavonoids and antioxidant activity of common brown beans (Phaseolus vulgaris L.) before and after cooking. J. Nutr. Food Sci. 2016, 6, 1-7. [CrossRef]

84. Díaz, A.M.; Caldas, G.V.; Blair, M.W. Concentrations of condensed tannins and anthocyanins in common bean seed coats. Food Res. Int. 2010, 43, 595-601. [CrossRef]

85. Xu, B.; Chang, S.K. Total phenolic, phenolic acid, anthocyanin, flavan-3-ol, and flavonol profiles and antioxidant properties of pinto and black beans (Phaseolus vulgaris L.) as affected by thermal processing. J. Agric. Food Chem. 2009, 57, 4754-4764. [CrossRef] [PubMed]

86. Chung, H.J.; Liu, Q.; Pauls, K.P.; Fan, M.Z.; Yada, R. In vitro starch digestibility, expected glycemic index and some physicochemical properties of starch and flour from common bean (Phaseolus vulgaris L.) varieties grown in Canada. Food Res. Int. 2008, 41, 869-875. [CrossRef] 
87. Romani, A.; Vignolini, P.; Galardi, C.; Mulinacci, N.; Benedettelli, S.; Heimler, D. Germplasm characterization of Zolfino landraces (Phaseolus vulgaris L.) by flavonoid content. J. Agric. Food Chem. 2004, 52, 3838-3842. [CrossRef] [PubMed]

88. Jenkins, A.L. The glycemic index: Looking back 25 years. Cereal Foods World 2007, 52, 50-53. [CrossRef]

89. Oomah, B.D.; Cardador-Martinez, A.; Loarca-Piña, G. Phenolics and antioxidative activities in common beans (Phaseolus vulgaris L). J. Sci. Food Agric. 2005, 85, 935-942. [CrossRef]

90. Cardador-Martínez, A.; Albores, A.; Bah, M.; Calderón-Salinas, V.; Castaño-Tostado, E.; Guevara-González, R.; Shimada-Miyasaka, A.; Loarca-Piña, G. Relationship among antimutagenic, antioxidant and enzymatic activities of methanolic extract from common beans (Phaseolus vulgaris $\mathrm{L}$ ). Plant Foods Hum. Nutr. 2006, 61, 161-168. [CrossRef] [PubMed]

91. $\mathrm{Xu}, \mathrm{B}$;; Chang, S.K. Total phenolics, phenolic acids, isoflavones, and anthocyanins and antioxidant properties of yellow and black soybeans as affected by thermal processing. J. Agric. Food Chem. 2008, 56, 7165-7175. [CrossRef] [PubMed]

92. Karaś, M.; Jakubczyk, A.; Szymanowska, U.; Materska, M.; Zielińska, E. Antioxidant activity of protein hydrolysates from raw and heat-treated yellow string beans (Phaseolus vulgaris L.). Acta Sci. Pol. Technol. Aliment. 2014, 13, 385-391. [CrossRef] [PubMed]

93. Frassinetti, S.; Gabriele, M.; Caltavuturo, L.; Longo, V.; Pucci, L. Antimutagenic and antioxidant activity of a selected lectin-free common bean (Phaseolus vulgaris L.) in two cell-based models. Plant Foods Hum. Nutr. 2015, 70, 35-41. [CrossRef] [PubMed]

94. Venkateswaran, S.; Pari, L. Antioxidant effect of Phaseolus vulgaris in streptozotocin-induced diabetic rats. Asia Pac. J. Clin. Nutr. 2002, 11, 206-209. [CrossRef] [PubMed]

95. Amarowicz, R.; Dykes, G.A.; Pegg, R.B. Antibacterial activity of tannin constituents from Phaseolus vulgaris, Fagoypyrum esculentum, Corylus avellana and Juglans nigra. Fitoterapia 2008, 79, 217-219. [CrossRef] [PubMed]

96. Lara-Díaz, V.J.; Gaytán-Ramos, A.A.; Dávalos-Balderas, A.J.; Santos-Guzmán, J.; Mata-Cárdenas, B.D.; Vargas-Villarreal, J.; Barbosa-Quintana, A.; Sanson, M.; López-Reyes, A.G.; Moreno-Cuevas, J.E. Microbiological and toxicological effects of Perla black bean (Phaseolus vulgaris L.) extracts: In vitro and in vivo studies. Basic Clin. Pharmacol. Toxicol. 2009, 104, 81-86. [CrossRef] [PubMed]

97. Ranilla, L.G.; Kwon, Y.I.; Genovese, M.I.; Lajolo, F.M.; Shetty, K. Effect of thermal treatment on phenolic compounds and functionality linked to type 2 diabetes and hypertension management of Peruvian and Brazilian bean cultivars (Phaseolus vulgaris L.) using in vitro methods. J. Food Biochem. 2010, 34, 329-355. [CrossRef]

98. Nilsson, A.; Johansson, E.; Ekström, L.; Björck, I. Effects of a brown beans evening meal on metabolic risk markers and appetite-regulating hormones at a subsequent standardized breakfast: A randomized cross-over study. PLoS ONE 2013, 8, e59985. [CrossRef] [PubMed]

99. Rondanelli, M.; Giacosa, A.; Orsini, F.; Opizzi, A.; Villani, S. Appetite control and glycemia reduction in overweight subjects treated with a combination of two highly standardized extracts from Phaseolus vulgaris and Cynara scolymus. Phytother. Res. 2011, 25, 1275-1282. [PubMed]

100. Okada, Y.; Okada, M.; Sagesaka, Y. Screening of dried plant seed extracts for adiponectin production activity and tumor necrosis factor-alpha inhibitory activity on 3T3-L1 adipocytes. Plant Foods Hum. Nutr. 2010, 65, 225-232. [CrossRef] [PubMed]

101. Mojica, L.; Meyer, A.; Berhow, M.A.; González de Mejía, E. Bean cultivars (Phaseolus vulgaris L.) have similar high antioxidant capacity, in vitro inhibition of $\alpha$-amylase and $\alpha$-glucosidase while diverse phenolic composition and concentration. Food Res. Int. 2015, 69, 38-48. [CrossRef]

102. Oseguera-Toledo, M.E.; de Mejia, E.G.; Dia, V.P.; Amaya-Llano, S.L. Common bean (Phaseolus vulgaris L.) hydrolysates inhibit inflammation in LPS-induced macrophages through suppression of NF-kB pathways. Food Chem. 2011, 127, 1175-1185. [CrossRef] [PubMed]

103. Gupta, A.; Agarwal, N.K.; Byadgi, P.S. Clinical assessment of dietary interventions and lifestyle modifications in Madhumeha (type-2 diabetes mellitus). Ayu 2014, 35, 391-397. [PubMed]

104. Kyznietsova, M.Y.; Halenova, T.I.; Savchuk, O.M.; Vereschaka, V.V.; Ostapchenko, L.I. Carbohydrate metabolism in type 1 diabetic rats under the conditions of the kidney bean pods aqueous extract application. Fiziolohichny̆ Zhurnal 2015, 61, 96-103. [CrossRef] [PubMed]

105. Pari, L.; Venkateswaran, S. Effect of an aqueous extract of Phaseolus vulgaris on plasma insulin and hepatic key enzymes of glucose metabolism in experimental diabetes. Pharmazie 2003, 58, 916-919. [PubMed] 
106. Spadafranca, A.; Rinelli, S.; Riva, A.; Morazzoni, P.; Magni, P.; Bertoli, S.; Battezzati, A. Phaseolus vulgaris extract affects glycometabolic and appetite control in healthy human subjects. Br. J. Nutr. 2013, 109, 1789-1795. [CrossRef] [PubMed]

107. Thompson, M.D.; Mensack, M.M.; Jiang, W.; Zhu, Z.; Lewis, M.R.; McGinley, J.N.; Brick, M.A.; Thompson, H.J. Cell signaling pathways associated with a reduction in mammary cancer burden by dietary common bean (Phaseolus vulgaris L.). Carcinogenesis 2012, 33, 226-232. [CrossRef] [PubMed]

108. Helmstädter, A. Beans and diabetes: Phaseolus vulgaris preparations as antihyperglycemic agents. J. Med. Food 2010, 13, 251-254. [CrossRef] [PubMed]

109. Tormo, M.A.; Gil-Exojo, I.; Romero de Tejada, A.; Campillo, J.E. Hypoglycaemic and anorexigenic activities of an alpha-amylase inhibitor from white kidney beans (Phaseolus vulgaris) in Wistar rats. Br. J. Nutr. 2004, 92, 785-790. [CrossRef] [PubMed]

110. Tormo, M.A.; Gil-Exojo, I.; Romero de Tejada, A.; Campillo, J.E. White bean amylase inhibitor administered orally reduces glycaemia in type 2 diabetic rats. Br. J. Nutr. 2006, 96, 539-544. [PubMed]

111. Balestri, F.; Rotondo, R.; Moschini, R.; Pellegrino, M.; Cappiello, M.; Barracco, V.; Misuri, L.; Sorce, C.; Andreucci, A.; Del-Corso, A.; et al. Zolfino landrace (Phaseolus vulgaris L.) from Pratomagno: General and specific features of a functional food. Food Nutr. Res. 2016, 60, 31792-31803. [CrossRef] [PubMed]

112. Pérez-Ramírez, I.F.; Becerril-Ocampo, L.J.; Reynoso-Camacho, R.; Herrera, M.D.; Guzmán-Maldonado, S.H.; Cruz-Bravo, R.K. Cookies elaborated with oat and common bean flours improved serum markers in diabetic rats. J. Sci. Food Agric. 2017. [CrossRef] [PubMed]

113. Ye, X.Y.; Ng, T.B.; Tsang, P.W.; Wang, J. Isolation of a homodimeric lectin with antifungal and antiviral activities from red kidney bean (Phaseolus vulgaris) seeds. J. Protein Chem. 2001, 20, 367-375. [CrossRef] [PubMed]

114. Daniell, E.L.; Ryan, E.P.; Brick, M.A.; Thompson, H.J. Dietary dry bean effects on hepatic expression of stress and toxicity-related genes in rats. Br. J. Nutr. 2012, 108, 37-45. [CrossRef] [PubMed]

115. García-Lafuente, A.; Moro, C.; Manchón, N.; Gonzalo-Ruiz, A.; Villares, A.; Guillamón, E.; Rostagno, M.; Mateo-Vivaracho, L. In vitro anti-inflammatory activity of phenolic-rich extracts from white and red common beans. Food Chem. 2014, 161, 216-223. [CrossRef] [PubMed]

116. Zhu, Z.; Jiang, W.; Thompson, H.J. Edible dry bean consumption (Phaseolus vulgaris L.) modulates cardiovascular risk factors and diet-induced obesity in rats and mice. Br. J. Nutr. 2012, 108, 66-73. [CrossRef] [PubMed]

117. Oomah, B.D.; Corbé, A.; Balasubramanian, P. Antioxidant and anti-inflammatory activities of bean (Phaseolus vulgaris L.) hulls. J. Agric. Food Chem. 2010, 58, 8225-8230. [CrossRef] [PubMed]

118. Gabriele, M.; Pucci, L.; La Marca, M.; Lucchesi, D.; Della Croce, C.M.; Longo, V.; Lubrano, V. A fermented bean flour extract down-regulates LOX-1, CHOP, and ICAM-1 in HMEC-1 stimulated by ox-LDL. Cell Mol. Biol. Lett. 2016, 21, 10-21. [CrossRef] [PubMed]

119. Reverri, E.J.; Randolph, J.M.; Steinberg, F.M.; Kappagoda, C.T.; Edirisinghe, I.; Burton-Freeman, B.M. Black beans, fiber, and antioxidant capacity pilot study: Examination of whole foods vs. functional components on postprandial metabolic, oxidative stress, and inflammation in adults with metabolic syndrome. Nutrients 2015, 7, 6139-6154. [CrossRef] [PubMed]

120. Monk, J.M.; Lepp, D.; Wu, W.; Pauls, K.P.; Robinson, L.E.; Power, K.A. Navy and black bean supplementation primes the colonic mucosal microenvironment to improve gut health. J. Nutr. Biochem. 2017, 49, 89-100. [CrossRef] [PubMed]

121. Ombra, M.N.; d'Acierno, A.; Nazzaro, F.; Riccardi, R.; Spigno, P.; Zaccardelli, M.; Pane, C.; Maione, M.; Fratianni, F. Phenolic composition and antioxidant and antiproliferative activities of the extracts of twelve common bean (Phaseolus vulgaris L.) endemic ecotypes of southern Italy before and after cooking. Oxid. Med. Cell. Longev. 2016, 2016, 1398298. [CrossRef] [PubMed]

122. Guajardo-Flores, D.; Serna-Saldívar, S.O.; Gutiérrez-Uribe, J.A. Evaluation of the antioxidant and antiproliferative activities of extracted saponins and flavonols from germinated black beans (Phaseolus vulgaris L.). Food Chem. 2013, 141, 1497-1503. [CrossRef] [PubMed]

123. Venkateswaran, S.; Pari, L.; Saravanan, G. Effect of Phaseolus vulgaris on circulatory antioxidants and lipids in rats with streptozotocin-induced diabetes. J. Med. Food 2002, 5, 97-103. [CrossRef] [PubMed] 
124. Mendoza-Sánchez, M.; Guevara-González, R.G.; Castaño-Tostado, E.; Mercado-Silva, E.M.; Acosta-Gallegos, J.A.; Rocha-Guzmán, N.E.; Reynoso-Camacho, R. Effect of chemical stress on germination of cv Dalia bean (Phaseolus vularis L.) as an alternative to increase antioxidant and nutraceutical compounds in sprouts. Food Chem. 2016, 212, 128-137. [CrossRef] [PubMed]

125. Ranilla, L.G.; Genovese, M.I.; Lajolo, F.M. Effect of different cooking conditions on phenolic compounds and antioxidant capacity of some selected Brazilian bean (Phaseolus vulgaris L.) cultivars. J. Agric. Food Chem. 2009, 57, 5734-5742. [CrossRef] [PubMed]

126. Pereira, M.P.; Tavano, O.L. Use of different spices as potential natural antioxidant additives on cooked beans (Phaseolus vulgaris). Increase of DPPH radical scavenging activity and total phenolic content. Plant Foods Hum. Nutr. 2014, 69, 337-343. [CrossRef] [PubMed]

127. Hernández-Salazar, M.; Osorio-Diaz, P.; Loarca-Piña, G.; Reynoso-Camacho, R.; Tovar, J.; Bello-Pérez, L.A. In vitro fermentability and antioxidant capacity of the indigestible fraction of cooked black beans (Phaseolus vulgaris L.), lentils (Lens culinaris L.) and chickpeas (Cicer arietinum L.). J. Sci. Food Agric. 2010, 90, 1417-1422. [CrossRef] [PubMed]

128. Aguilera, Y.; Liébana, R.; Herrera, T.; Rebollo-Hernanz, M.; Sanchez-Puelles, C.; Benítez, V.; Martín-Cabrejas, M.A. Effect of illumination on the content of melatonin, phenolic compounds, and antioxidant activity during germination of lentils (Lens culinaris L.) and kidney beans (Phaseolus vulgaris L.). J. Agric. Food Chem. 2014, 62, 10736-10743. [CrossRef] [PubMed]

129. Luzardo-Ocampo, I.; Campos-Vega, R.; Gaytán-Martínez, M.; Preciado-Ortiz, R.; Mendoza, S.; Loarca-Piña, G. Bioaccessibility and antioxidant activity of free phenolic compounds and oligosaccharides from corn (Zea mays L.) and common bean (Phaseolus vulgaris L.) chips during in vitro gastrointestinal digestion and simulated colonic fermentation. Food Res. Int. 2017, 100, 304-311. [CrossRef] [PubMed]

130. Thompson, M.D.; Thompson, H.J.; Brick, M.A.; McGinley, J.N.; Jiang, W.; Zhu, Z.; Wolfe, P. Mechanisms associated with dose-dependent inhibition of rat mammary carcinogenesis by dry bean (Phaseolus vulgaris, L.). J. Nutr. 2008, 138, 2091-2097. [CrossRef] [PubMed]

131. Thompson, S.V.; Winham, D.M.; Hutchins, A.M. Bean and rice meals reduce postprandial glycemic response in adults with type 2 diabetes: A cross-over study. Nutr. J. 2012, 11, 23-30. [CrossRef] [PubMed]

132. Mensack, M.M.; McGinley, J.N.; Thompson, H.J. Metabolomic analysis of the effects of edible dry beans (Phaseolus vulgaris L.) on tissue lipid metabolism and carcinogenesis in rats. Br. J. Nutr. 2012, 108, 155-165. [CrossRef] [PubMed]

133. Haydé, V.C.; Ramón, G.G.; Lorenzo, G.O.; Dave, O.B.; Rosalía, R.C.; Paul, W.; Guadalupe, L.P. Non-digestible fraction of beans (Phaseolus vulgaris L.) modulates signaling pathway genes at an early stage of colon cancer in Sprague-Dawley rats. Br. J. Nutr. 2012, 108, 145-154. [CrossRef] [PubMed]

134. Vergara-Castañeda, H.A.; Guevara-González, R.G.; Ramos-Gómez, M.; Reynoso-Camacho, R.; Guzmán-Maldonado, H.; Feregrino-Pérez, A.A.; Oomah, B.D.; Loarca-Piña, G. Non-digestible fraction of cooked bean (Phaseolus vulgaris L.) cultivar Bayo Madero suppresses colonic aberrant crypt foci in azoxymethane-induced rats. Food Funct. 2010, 1, 294-300. [CrossRef] [PubMed]

135. Feregrino-Pérez, A.A.; Berumen, L.C.; García-Alcocer, G.; Guevara-Gonzalez, R.G.; Ramos-Gomez, M.; Reynoso-Camacho, R.; Acosta-Gallegos, J.A.; Loarca-Piña, G. Composition and chemopreventive effect of polysaccharides from common beans (Phaseolus vulgaris L.) on azoxymethane-induced colon cancer. J. Agric. Food Chem. 2008, 56, 8737-8744. [CrossRef] [PubMed]

136. Feregrino-Perez, A.A.; Piñol-Felis, C.; Gomez-Arbones, X.; Guevara-González, R.G.; Campos-Vega, R.; Acosta-Gallegos, J.; Loarca-Piña, G. A non-digestible fraction of the common bean (Phaseolus vulgaris L.) induces cell cycle arrest and apoptosis during early carcinogenesis. Plant Foods Hum. Nutr. 2014, 69, 248-254. [CrossRef] [PubMed]

137. Campos-Vega, R.; García-Gasca, T.; Guevara-Gonzalez, R.; Ramos-Gomez, M.; Oomah, B.D.; Loarca-Piña, G. Human gut flora-fermented non-digestible fraction from cooked bean (Phaseolus vulgaris L.) modifies protein expression associated with apoptosis, cell cycle arrest, and proliferation in human adenocarcinoma colon cancer cells. J. Agric. Food Chem. 2012, 60, 12443-12450. [CrossRef] [PubMed]

138. Nakanishi, S.; Kataoka, K.; Kuwahara, T.; Ohnishi, Y. Effects of high amylose maize starch and Clostridium butyricum on metabolism in colonic microbiota and formation of azoxymethane-induced aberrant crypt foci in the rat colon. Microbiol. Immunol. 2003, 47, 951-958. [CrossRef] [PubMed] 
139. Cruz-Bravo, R.K.; Guevara-Gonzalez, R.; Ramos-Gomez, M.; Garcia-Gasca, T.; Campos-Vega, R.; Oomah, B.D.; Loarca-Piña, G. Fermented nondigestible fraction from common bean (Phaseolus vulgaris L.) cultivar Negro 8025 modulates HT-29 cell behavior. J. Food Sci. 2011, 76, 41-47.

140. Chávez-Santoscoy, R.A.; Gutiérrez-Uribe, J.A.; Serna-Saldívar, S.O. Effect of flavonoids and saponins extracted from black bean (Phaseolus vulgaris L.) seed coats as cholesterol micelle disruptors. Plant Foods Hum. Nutr. 2013, 68, 416-423. [CrossRef] [PubMed]

141. Sidorova, Y.; Shipelin, V.; Mazo, V.; Zorin, S.; Petrov, N.; Kochetkova, A. Hypoglycemic and hypolipidemic effect of Vaccinium myrtillus L. leaf and Phaseolus vulgaris L. seed coat extracts in diabetic rats. Nutrition 2017, 41, 107-112. [CrossRef] [PubMed]

142. Pari, L.; Venkateswaran, S. Protective role of Phaseolus vulgaris on changes in the fatty acid composition in experimental diabetes. J. Med. Food. 2004, 7, 204-209. [CrossRef] [PubMed]

143. Loi, B.; Fantini, N.; Colombo, G.; Gessa, G.L.; Riva, A.; Bombardelli, E.; Morazzoni, P.; Carai, M.A. Reducing effect of an extract of Phaseolus vulgaris on food intake in micefocus on highly palatable foods. Fitoterapia 2013, 85, 14-19. [CrossRef] [PubMed]

144. Zaru, A.; Maccioni, P.; Riva, A.; Morazzoni, P.; Bombardelli, E.; Gessa, G.L.; Carai, M.A.; Colombo, G. Reducing effect of a combination of Phaseolus vulgaris and Cynara scolymus extracts on operant self-administration of a chocolate-flavored beverage in rats. Phytother. Res. 2013, 27, 944-947. [CrossRef] [PubMed]

145. Maccioni, P.; Colombo, G.; Riva, A.; Morazzoni, P.; Bombardelli, E.; Gessa, G.L.; Carai, M.A. Reducing effect of a Phaseolus vulgaris dry extract on operant self-administration of a chocolate-flavored beverage in rats. Br. J. Nutr. 2010, 104, 624-628. [CrossRef] [PubMed]

146. Fantini, N.; Cabras, C.; Lobina, C.; Colombo, G.; Gessa, G.L.; Riva, A.; Donzelli, F.; Morazzoni, P.; Bombardelli, E.; Carai, M.A. Reducing effect of a Phaseolus vulgaris dry extract on food intake, body weight, and glycemia in rats. J. Agric. Food Chem. 2009, 57, 9316-9323. [CrossRef] [PubMed]

147. Carai, M.A.; Fantini, N.; Loi, B.; Colombo, G.; Gessa, G.L.; Riva, A.; Bombardelli, E.; Morazzoni, P. Multiple cycles of repeated treatments with a Phaseolus vulgaris dry extract reduce food intake and body weight in obese rats. Br. J. Nutr. 2011, 106, 762-768. [CrossRef] [PubMed]

148. Loi, B.; Fantini, N.; Colombo, G.; Gessa, G.L.; Riva, A.; Bombardelli, E.; Morazzoni, P.; Carai, M.A. Reducing effect of a combination of Phaseolus vulgaris and Cynara scolymus extracts on food intake and glycemia in rats. Phytother. Res. 2013, 27, 258-263. [CrossRef] [PubMed]

149. Hernández-Saavedra, D.; Mendoza-Sánchez, M.; Hernández-Montiel, H.L.; Guzmán-Maldonado, H.S.; Loarca-Piña, G.F.; Salgado, L.M.; Reynoso-Camacho, R. Cooked common beans (Phaseolus vulgaris) protect against $\beta$-cell damage in streptozotocin-induced diabetic rats. Plant Foods Hum. Nutr. 2013, 68, $207-212$. [CrossRef] [PubMed]

150. Venn, B.J.; Mann, J.I. Cereal grains, legumes and diabetes. Eur. J. Clin. Nutr. 2004, 58, 1443-1461. [CrossRef] [PubMed]

151. Campos-Vega., R.; Loarca-Pina, G.; Oomah, B.D. Minor components of pulses and their potential impact on human health. Food Res. Int. 2010, 43, 461-582. [CrossRef]

152. Oseguera-Toledo, M.E.; Gonzalez de Mejia, E.; Amaya-Llano, S.L. Hard-to-cook bean (Phaseolus vulgaris L.) proteins hydrolyzed by alcalase and bromelain produced bioactive peptide fractions that inhibit targets of type-2 diabetes and oxidative stress. Food Res. Int. 2015, 76, 839-851. [CrossRef] [PubMed]

153. Mojica, L.; de Mejía, E.G. Optimization of enzymatic production of anti-diabetic peptides from black bean (Phaseolus vulgaris L.) proteins, their characterization and biological potential. Food Funct. 2016, 7, 713-727. [CrossRef] [PubMed]

154. Roman-Ramos, R.; Flores-Saenz, F.J.; Alarcon, A. Anti-hyperglycemic effect of some edible plants. J. Ethnopharmacol. 1995, 48, 25-32. [CrossRef]

155. Villegas, R.; Gao, Y.T.; Yang, G.; Li, H.L.; Elasy, T.A.; Zheng, W. Legume and soy food intake and the incidence of type 2 diabetes in the Shanghai Women's Health Study. Am. J. Clin. Nutr. 2008, 87, 162-167. [PubMed]

156. Tang, G.Y.; Li, X.J.; Zhang, H.Y. Antidiabetic components contained in vegetables and legumes. Molecules 2008, 13, 1189-1194. [CrossRef] [PubMed]

157. Pi-Sunyer, F.X. Pathophysiology and long-term management of the metabolic syndrome. Obes. Res. 2004, 12, 174-180. [CrossRef] [PubMed] 
158. Finley, J.W.; Burrell, J.B.; Reeves, P.G. Pinto bean consumption changes SCFA profiles in fecal fermentations, bacterial populations of the lower bowel, and lipid profiles in blood of humans. J. Nutr. 2007, 137, 2391-2398. [PubMed]

159. Anderson, J.W.; Major, A.W. Pulses, and lipaemia, short- and long-term effects: Potential in the prevention of cardiovascular disease. Br. J. Nutr. 2002, 88, 263-271. [CrossRef] [PubMed]

160. Winham, D.M.; Hutchins, M.H. Baked beans consumption reduces serum cholesterol in hypercholesterolemic adults. Nutr. Res. 2007, 27, 380-386. [CrossRef]

161. Shutler, S.M.; Bircher, G.M.; Tredger, J.A.; Morgan, L.M.; Walker, A.F.; Low, A.G. The effect of daily baked bean (Phaseolus vulgaris) consumption on the plasma lipid levels of young, normocholesterolemic men. Br. J. Nutr. 1989, 61, 257-265. [CrossRef] [PubMed]

162. Anderson, J.W.; Story, L.; Sieling, B.; Chen, W.L.; Petro, M.S.; Story, J. Hypocholesterolemic effects of bean intake for hypercholesterolemic men. Am. J. Clin. Nutr. 1990, 40, 1146-1155.

163. Maruyama, C.; Araki, R.; Kawamura, M.; Kondo, N.; Kigawa, M.; Kawai, Y. Azuki bean juice lowers serum triglyceride concentrations in healthy young women. J. Clin. Biochem. Nutr. 2008, 43, 19-25. [CrossRef] [PubMed]

164. Bazzano, L.H.J.; Ogden, L.G.; Loria, C.; Vupputuri, S.; Myers, L.; Whelton, P.K. Legume consumption and risk of coronary heart disease in US men and women: NHANES I Epidemiologic Follow-up Study. Arch. Int. Med. 2001, 161, 2573-2578. [CrossRef]

165. Chavez-Santoscoy, R.A.; Gutierrez-Uribe, J.A.; Granados, O.; Torre-Villalvazo, I.; Serna-Saldivar, S.O.; Torres, N.; Palacios-González, B.; Tovar, A.R. Flavonoids and saponins extracted from black bean (Phaseolus vulgaris L.) seed coats modulate lipid metabolism and biliary cholesterol secretion in C57BL/6 mice. Br. J. Nutr. 2014, 112, 886-899. [CrossRef] [PubMed]

166. Wu, P.; Liu, S.; Su, J.; Chen, J.; Li, L.; Zhang, R.; Chen, T. Apoptosis triggered by isoquercitrin in bladder cancer cells by activating the AMPK-activated protein kinase pathway. Food Funct. 2017. [CrossRef] [PubMed]

167. Chatterjee, A.; Ronghe, A.; Padhye, S.B.; Spade, D.A.; Bhat, N.K.; Bhat, H.K. Antioxidant activities of novel resveratrol analogs in breast cancer. J. Biochem. Mol. Toxicol. 2017. [CrossRef] [PubMed]

168. Correa, P. Epidemiological correlations between diet and cancer frequency. Cancer Res. 1981, 41, 3685-3689. [PubMed]

169. Kolonel, L.N.; Hankin, J.H.; Whittemore, A.S.; Wu, A.H.; Gallagher, R.P.; Wilkens, L.R.; John, E.M.; Howe, G.R.; Dreon, D.M.; West, D.W.; et al. Vegetables, fruits, legumes and prostate cancer: A multiethnic case-control study. Cancer Epidemiol. Biomark. Prev. 2000, 9, 795-804.

170. Thompson, M.D.; Brick, M.A.; McGinley, J.N.; Thompson, H.J. Chemical composition and mammary cancer inhibitory activity of dry beans. Crop Sci. 2009, 49, 179-186. [CrossRef]

171. Hangen, L.A.; Bennink, M.R. Consumption of black beans and navy beans (Phaseolus vulgaris) reduced azoxymethane-induced colon cancer in rats. Nutr. Cancer 2003, 44, 60-65.

172. Williams, G.M.; Iatropoulos, M.J.; Jeffrey, A.M. Anticarcinogenicity of monocyclic phenolic compounds. Eur. J. Cancer Prev. 2002, 11, 101-107.

173. Sabater, V.M.; Kuilman-Wahls, M.E.M.; Fink-Gremmels, J. Inhibition of aflatoxin B1 mutagenicity by cyclopiazonic acid in the presence of human liver preparations. Toxicol. Lett. 2003, 143, 291-299. [CrossRef]

174. De Flora, S. Mechanisms of inhibitors of mutagenesis and carcinogenesis. Mutat. Res. 1998, 402, 151-158. [CrossRef]

(C) 2017 by the authors. Licensee MDPI, Basel, Switzerland. This article is an open access article distributed under the terms and conditions of the Creative Commons Attribution (CC BY) license (http:/ / creativecommons.org/licenses/by/4.0/). 